\title{
Non-indigenous plant species and their ecological range in Central European pine (Pinus sylvestris L.) forests
}

\author{
Stefan ZERBE*, Petra WIRTH \\ Institute of Ecology, Technical University Berlin, Rothenburgstraße 12, 12165 Berlin, Germany
}

(Received 21 January 2005; accepted 30 June 2005)

\begin{abstract}
In this study, forest ecosystems were analysed with regard to the occurrence and ecological range of non-indigenous plant species. Pine forests in the NE German lowland, which naturally and anthropogenically occur on a broad range of different sites, were taken as an example. The analysis is based on a data set of about 2300 vegetation plots. The ecological range was assessed applying Ellenberg's ecological indicator values. Out of a total of 362 taxa recorded in the pine forests, only 12 non-indigenous species, including trees, shrubs, annual and perennial herbs, and one bryophyte were found. They commonly grow on sites with relatively high nitrogen availability and soil reaction values. Most species are native to North America. Taking into account that a high proportion of the investigated pine forests is of anthropogenic origin and will naturally develop towards broad-leaved forests with beech and oak, it is hypothesised that most of the observed invasions are reversible.
\end{abstract}

Ellenberg indicator values / forest development / human impact / nitrogen availability / plant invasions

Résumé - Espèces non indigènes et leur habitat écologique dans les forêts de pins (Pinus sylvestris L.) de l'Europe Centrale. Dans cette étude, les écosystèmes forestiers ont été analysés eu égard à l'occurrence et à l'habitat écologique des espèces de plantes non-indigènes. Les forêts de pins dans les plaines du NE de l'Allemagne, dans lesquelles il existe naturellement et anthropogénétiquement une large gamme de sites différents, ont été prises en exemple. L'analyse s'appuie sur un ensemble de données d'environ 2300 placeaux. La gamme écologique a été établie en ayant recours aux indicateurs écologiques d'Ellenberg. Sur un total de 362 taxa notés dans les forêts de pins, seulement 12 espèces non indigènes ont été trouvées en incluant les arbres, les buissons, les herbacées annuelles et pérennes. Un seul bryophyte a été identifié. Elles se développent communément sur les sites présentant une disponibilité en azote et une réaction élevée à l'acide. Beaucoup d'espèces sont originaires d'Amérique du Nord. Prenant en compte le fait qu'une forte proportion de forêts de pins étudiées a une origine anthropogène et le fait que naturellement se développeront des forêts feuillues avec le hêtre et le chêne, il est fait l'hypothèse que la plus grande partie de ces invasions sont réversibles.

valeurs indicatrices d'Ellenberg / développement de la forêt / impact humain / disponibilité en azote / invasions de plantes

Nomenclature: [68] for vascular plants, [17] for bryophytes, and [67] for lichens.

\section{INTRODUCTION}

Detailed knowledge on the biology, ecology, and management of non-indigenous plant species is continuously increasing due to numerous investigations throughout the world. Among the driving forces for this intense research is the fact that invasions by non-indigenous organisms and the subsequent biodiversity loss is recognized as one of the biggest global environmental problems of our time [54, 64]. Additionally, the costs related to biological invasions, for example for the management of established and invasive non-indigenous species, can be considerably high for society (e.g. [63]).

In Central Europe, invasions by non-indigenous plants are recorded and investigated along the whole range from anthropogenically strongly altered towards natural ecosystems [34]. Thus, for example, settlements (e.g. $[52,72])$, grassland (e.g.
[65]), fields (e.g. [23]), and mires e.g. [56] have been studied with regard to plant invasions, both concentrating on invasive species as well as invaded habitats. Compared to these non-forest habitats, there are much less studies on plant invasions in Central European forest ecosystems. For example, the invasion of the herb Impatiens parviflora, which has its origin in East Asia, is well documented and analysed, focusing on the species biology, ecology, and the forest communities, which are invaded [61]. Additionally, non-indigenous tree species, such as the North American Prunus serotina [59], Pseudotsuga menziesii [26], and Robinia pseudoacacia [25, 40], which are invaders in Central European woodland, have been investigated in detail with regard to their biology and ecology. Lohmeyer and Sukopp [40] give a survey on non-indigenous plant species in Central Europe, which are invasive to natural habitats (so-called agriophytes), also including forest ecosystems. The ecological range

* Corresponding author: Stefan.Zerbe@TU-Berlin.de 


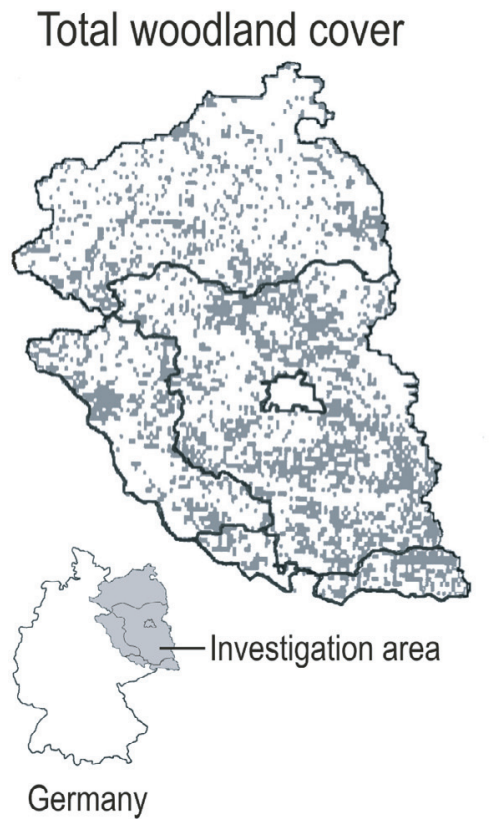

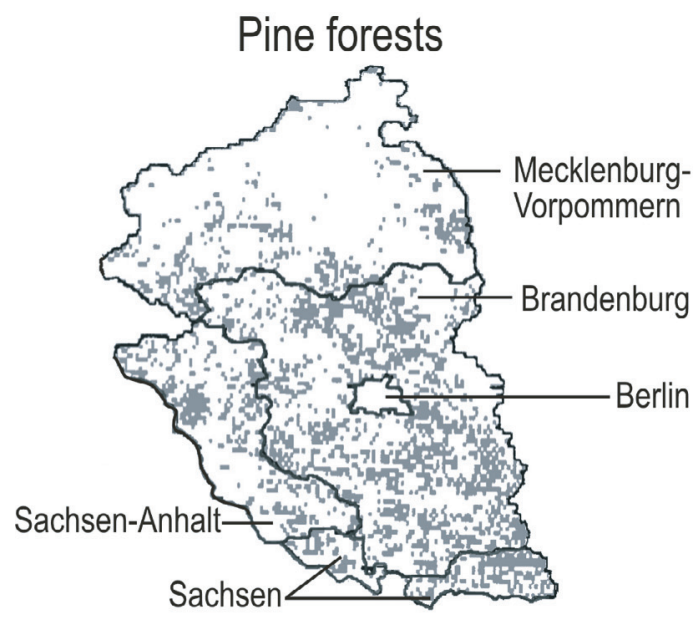

Figure 1. Pine dominated woodland in the study area of the north-eastern German lowland in comparison with the total woodland cover (according to data from Hofmann [22] with no data for Berlin given). of these species can be derived from the plant communities in which they occur.

Up to now, there is a lack of comprehensive studies on plant invasions in forests, which aim at a quantitative and qualitative ecological analysis of non-indigenous species based on large vegetation data sets. We aim to fill this gap with a focus on Central European pine forests. Naturally, Scots pine (Pinus sylvestris) only dominates the tree layer in certain regions or on certain sites in Central Europe where local climate and/or soil conditions are not favourable for a dominance of broad-leaved trees like beech (Fagus sylvatica) or oak (Quercus petraea and Quercus robur; [14]). However, pine has become one of the most important tree species in Central European lowlands due to large-scaled plantations since the end of the 18th century [70]. After a long period of forest destruction as a consequence of over-utilisation of forests and forest sites (e.g. by timber cutting, forest pasture, litter gathering, charcoal production, operation of forest glassworks), pine was particularly planted on sites with sandy soils $[19,39,45,55]$.

This study is based on 2289 phytosociological vegetation plots from anthropogenic and natural pine forests, which have been carried out by numerous authors. Pine forest communities are differentiated on the basis of the occurring species using a cluster analysis and ecologically characterized employing the ecological indicator values for Central European plant species from Ellenberg et al. [15]. The following hypotheses are addressed in this study: (1) only few non-indigenous plant species occur in forest ecosystems, and (2) there are specific site preferences (e.g. nutrient and water supply of the soil) of the non-indigenous species, which occur in pine forests. Additionally, human impact on the forests and forest sites is discussed with regard to favouring the establishment of non-indigenous species in forests. Non-indigenous plants are meant here as those species that have been introduced by man since $1500 \mathrm{AD}$ (usually termed "neophytes"; for the history of this term see [34]). From our results and the present knowledge on the devel- opment of anthropogenic pine towards natural forests, predictions are made with regard to the reversibility and irreversibility of the recorded plant invasions.

\section{STUDY AREA AND DATABASE}

The study area is the North-eastern German lowland including the federal states Mecklenburg-Vorpommern, Brandenburg, Berlin, the North-western part of Sachsen-Anhalt, and the Northern part of Sachsen (Fig. 1). The geology as well as the climate is characterised by pronounced gradients from $\mathrm{N}$ to $\mathrm{S}$ and NW to SE. The geological and geomorphological characters of the NE German lowland were formed during the glacial periods. Whereas the more or less loamy soils of the young pleistocene sediments in the northern part of the study area are rich in nutrients, despite of the outwash plains with purely sandy soils, the older pleistocene sediments in the southern part bear nutrient poor sandy soils [57]. The climate varies from oceanic at and near the Baltic Sea coast to more continental in the SE of the study area $[20,50]$. Thus, the mean annual precipitation of more than $600 \mathrm{~mm}$ and the mean annual air temperature of $8.4{ }^{\circ} \mathrm{C}$ (city of Schwerin, period of measurement 19611990) in the NW of the study area are distinctly different from the mean annual precipitation of less than $500 \mathrm{~mm}$ and the mean annual air temperature of $8.7^{\circ} \mathrm{C}$ in the south-west (city of Magdeburg, period of measurement 1961-1990 [46]).

The vegetation database, compiled from about 60 different studies from different authors (list available from authors), covers pine dominated anthropogenic and natural forests within the whole range of the above described geological and climatic gradient of the study area. The sampling was carried out according to the method of Braun-Blanquet [6] and aimed at an ecological characterisation and/or comprehensive inventories of natural and anthropogenic forest vegetation of a certain region. Following the method of Braun-Blanquet [6], the data were 
recorded on randomly selected plots, homogeneous with regard to the site conditions and the stand structure. Consequently, specific sites like, for example, forest paths, forest edges, and clear-cuts were excluded from these analyses. The age of the forest stands ranges from about 40 years to old-growth stands with more than 100 years.

\section{MATERIALS AND METHODS}

\subsection{Compilation of data}

The vegetation data were edited with the help of the program SORT [13]. Data based on different nomenclature were harmonised using the lists of Wisskirchen and Haeupler [68] for vascular plants, Frahm and Frey [17] for bryophytes, and Wirth [67] for lichens. Based on 2289 vegetation plots, the material was organised to reduce the heterogeneity of the data. Only plots fulfilling the following criteria were considered:

- Pinus sylvestris is the dominating tree species in the canopy (upper tree layer).

- Synoptic tables were not used because single vegetation plots could not be separated.

- Studies without any records of bryophytes were removed from the data set. As lichens do not commonly occur in pine forests (such as bryophytes), this was not done for samples without any lichen records.

\subsection{Data set properties}

In order to optimise the data structure, the following procedure was carried out (according to Diekmann et al. [10]). Only epigeic species were taken into consideration. Thus, epiphytic, epilithic, and epixylic species were removed from the data set. Fungi were neglected because there were only a few records, e.g. by Krausch [36]. Some species were determined at different taxonomical levels (e.g. Festuca ovina agg.), some at generic level (e.g. Cladonia sp.), and others at species or subspecies level (e.g. Silene latifolia ssp. alba). In general, all taxa were given names at the species level. However, some taxa were transferred to the generic level (e.g. Cladonia sp.) due to different determinations by different authors. According to recommendations of Wildi et al. [66], "difficult" (with regard to determination) taxa were combined (e.g. Galeopsis tetrahit/G. bifida and Viola reichenbachiana/V. riviniana) or denoted at the genus level (e.g. Rosa sp.) or as aggregate (e.g. Rubus fruticosus agg.).

Altogether 362 taxa were recorded, nine (about $2.5 \%$ ) only at the generic level. In total, 59 cryptogam species were recorded including mainly bryophytes.

\subsection{Cluster analysis}

For the statistical classification, all very rare species occurring only in five or less samples were removed from the data set. The data were classified with Ward's optimal agglomeration method [3] with the help of the statistical program SPSS [7]. Based on this hierarchical classification a synoptic table was created with 23 clusters or pine forest communities (Tab. I). In the table, all taxa were represented by their frequency (in \%), i.e. the number of sample plots in a cluster, in which a taxon occurred, related to the total number of sample plots in that cluster. In order to optimise the presentability of the table, only those species were considered which occurred with a frequency of more than $10 \%$ in at least one cluster. Thus, 258 taxa out of 362 are shown in Table I. Species, which reach the highest frequency values in a single cluster were considered as differentiating species of this community.

\subsection{Assessing the ecological range}

Environmental parameters (e.g. soil $\mathrm{pH}$ ) were only available for a very limited number of sample plots. Therefore, the environmental conditions of different communities (clusters) were assessed by means of ecological indicator values of the species present according to Ellenberg et al. [15] for vascular plants and Benkert et al. [4] for bryophytes (for the methodological approach see $[12,35])$. Indicator values for light $(\mathrm{L})$, continentality $(\mathrm{C})$, moisture $(\mathrm{M})$, soil reaction $(\mathrm{R})$, and nitrogen $(\mathrm{N})$ were computed. The values are expressed on a 1 to 9 scale, i.e. the higher the value, the higher the species' demand for the particular factor. As a first step, medians (not weighted) were calculated for the single plots. To avoid circular argumentation, the non-indigenous species were excluded from this calculation, which aims at an ecological assessment of the forest site conditions. Then, for each cluster and ecological factor, medians were calculated as an average value of all sample plots within the cluster. The values for each cluster were represented by Box-and-Whisker-Plots [41] with the minima and maxima given. The differences of mean indicator values between the clusters were tested for their statistical significance by the non-parametric rank sum test of Mann-Whitney [53] also using the SPSS software package.

\section{RESULTS}

\subsection{Ecological differentiation of the clusters (communities)}

The statistical classification resulted in 23 clusters or communities. A compilation is given in Table I. On the basis of the frequency of certain species or species groups within a single cluster, communities can be described, which correspond to different syntaxonomic levels of Braun-Blanquet's [6] classification system of Central European vegetation (for pine forests see $[5,19,43,47,69])$. For example, cluster 1 is characterized by the species Anthericum liliago, Artemisia campestris, Dianthus carthusianorum, Helichrysum arenarium, Peucedanum oreoselinum and others, which occur with a relatively low frequency and some exclusively in this cluster. Most of these species grow on sites, which are warm and dry throughout the summer season and rich in bases. Thus, cluster 1 corresponds to the Peucedano-Pinetum Matusz. 1962.

All 23 clusters can be separated in two community groups. Within the first group (clusters 1-12), species with a relatively high nutrient demand (particularly nitrogen) occur with frequencies up to $95 \%$. These are, for example, Epilobium angustifolium, Moehringia trinervia, Rubus fruticosus agg., Rubus idaeus, and Taraxacum officinale agg. On the contrary, species with a low nutrient demand, characteristic for acid and oligotrophic sites, are most frequent in the second group (clusters 13-23), e.g. Calluna vulgaris, Dicranum scoparium, and Vaccinium vitis-idaea.

Both, the mean Ellenberg indicator values for soil reaction $(\mathrm{R})$ and nitrogen $(\mathrm{N})$, reflect the floristic differentiation of the two cluster groups 1-12 and 13-23 (Fig. 2). Whereas in the first group all medians of the indicator values for soil reaction exceed the median 3.0, the medians of the second group range between 2.0 and 3.0, with the exception of cluster 18. Thus, the communities of the latter group grow on sites where soil is characterised by very low $\mathrm{pH}$ values. Significant differences between the medians appear within each of the two cluster 
Table I. Synoptic table of the clusters (communities) of pine forests in the lowland of NE Germany according to the Ward classification. Species occurrence is presented for vascular plants, bryophytes, and lichens with their frequency (in \%) in each cluster; species not achieving a frequency of more than $10 \%$ in at least one cluster were omitted; non-indigenous species in bold.

\begin{tabular}{lccccccccccccccccccccccccc}
\hline Cluster & 1 & 2 & 3 & 4 & 5 & 6 & 7 & 8 & 9 & 10 & 11 & 12 & 13 & 14 & 15 & 16 & 17 & 18 & 19 & 20 & 21 & 22 & 23 \\
Number of vegetation plots & 61 & 46 & 97 & 32 & 203 & 78 & 46 & 54 & 89 & 72 & 43 & 178 & 85 & 221 & 87 & 52 & 131 & 91 & 65 & 194 & 144 & 119 & 116 \\
\hline
\end{tabular}

Cluster differentiating species:

Agrostis vinealis

Artemisia campestris

Helichrysum arenarium

Anthericum liliago

Brachythecium explanatum

Sedum maximum

Peucedanum oreoselinum

Dianthus carthusianorum

Vincetoxicum hirundinaria

Euphrasia stricta

Hieracium lachenalii

Hieracium pilosella

Symphoricarpos albus

Acer platanoides

Tilia cordata

Crataegus monogyna

Acer pseudoplatanus

Cerastium holosteoides

Rosa canina

Hedera helix

Mahonia aquifolium

Vicia angustifolia

Ligustrum vulgare

Convallaria majalis

Vicia hirsuta

Platanthera bifolia

Conyza canadensis

Rubus saxatilis

Rhamnus cathartica

Cotoneaster spec.

Taxus baccata

Leontodon autumnalis

Rubus caesius

Amelanchier alnifolia

Acer negundo

Hypericum perforatum

Cirsium arvense

Plagiomnium affine

Inula conyzae

Valeriana officinalis

Pimpinella saxifraga

Ribes rubrum

$\begin{array}{llll}20 & 2 & . & . \\ 18 & . & . & . \\ 18 & . & 1 & . \\ 16 & . & . & . \\ 18 & . & . & . \\ 15 & . & . & . \\ 15 & . & . & . \\ 12 & . & . & 3 \\ 13 & 7 & . & . \\ 13 & . & . & .\end{array}$

$3 . \quad 1 \quad 6 \quad 2$

$\begin{array}{llllllllllllllll}18 & 89 & 1 & 25 & 11 & 3 & . & 4 & 2 & 15 & . & 5 & 2 & 3\end{array}$

$\begin{array}{llllllllllllllll}64 & 83 & 21 & 41 & 30 & 1 & . & 6 & 1 & . & 19 & 5 & 19 & 2\end{array}$

$\begin{array}{lllllllllllllllll}12 & 78 & 13 & 25 & . & . & . & . & 13\end{array}$

$\begin{array}{llllllllllllll}46 & 76 & 8 & 34 & 3 & . & 2 & 6 & 3 & 49 & 2 & 4 & 1 & 1\end{array}$

$\begin{array}{lllllllllllll}12 & 72 & 2 & 3 & 2 & . & . & 2 & . & 22 & 16 & 2\end{array}$

$\begin{array}{lllllllllllll}8 & 65 & 44 & 47 & 1 & . & 4 & 2 & . & 10 & 16 & 3\end{array}$

$\begin{array}{lllllllllll}15 & 63 & 5 & 22 & 1 & 9 & 6 & 5 & 57 & 2 & 11\end{array}$

$\begin{array}{llllllllllll}7 & 59 & 23 & 16 & 4 & . & 2 & 2 & 3 & 3 & & 2\end{array}$

$\begin{array}{llllllllllll}16 & 54 & 34 & 19 & 3 & 1 & 2 & . & 1 & . & . & 4\end{array}$

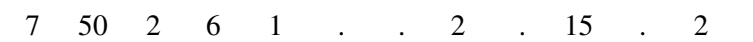

$\begin{array}{lllll}5 & 48 & 30 & 19\end{array}$

$\begin{array}{lll}12 & 46 & 1\end{array}$

$\begin{array}{llll}12 & 44 & 8 & 28\end{array}$

$\begin{array}{llll}21 & 41 & 1 & 9\end{array}$

$\begin{array}{llll}3 & 33 & 3 & 16\end{array}$

. 28

324

. 24

. 221

$\begin{array}{lllll}12 & 22 & 1 & . & 5\end{array}$

. 20.3

. 132

. 13

$\begin{array}{cccccccccccc}46 & 39 & 88 & 47 & 20 & 12 & 46 & 2 & . & 13 & 33 & 8 \\ . & 9 & 71 & 19 & . & . & 11 & . & . & 1 & . & 2 \\ 36 & 41 & 65 & 16 & 8 & 8 & 20 & . & . & 22 & . & 2\end{array}$

$\begin{array}{llllll}. & 4 & 4 & 4\end{array}$ 
Table I. Continued.

\begin{tabular}{|c|c|c|c|c|c|c|c|c|c|c|c|c|c|c|c|c|c|c|c|c|c|c|c|}
\hline Cluster & 1 & 2 & 3 & 4 & 5 & 6 & 7 & 8 & 9 & 10 & 11 & 12 & 13 & 14 & 15 & 16 & 17 & 18 & 19 & 20 & 21 & 22 & 23 \\
\hline Number of vegetation plots & 61 & 46 & 97 & 32 & 203 & 78 & 46 & 54 & 89 & 72 & 43 & 178 & 85 & 221 & 87 & 52 & 131 & 91 & 65 & 194 & 144 & 119 & 116 \\
\hline Galium pumilum & . & . & 33 & 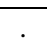 & 3 & . & 15 & . & . & . & . & . & . & . &. &. &. & . & . & . & . & . & . \\
\hline Campanula rotundifolia & 5 & 20 & 31 & 6 & 15 & . & 2 & . & . & 4 & 2 & 3 & 4 & 2 & . & . & 1 & 9 & 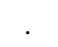 & 2 & 2 & 2 & 4 \\
\hline Asparagus officinalis & 7 & 11 & 29 & 13 & 1 & . & 11 & . & . & 3 & . & . & 1 & . & . & . & . & 8 & . & . & . & . & . \\
\hline Festuca rubra & 3 & 7 & 28 & 3 & 3 & . & 2 & . & . & 10 & . & 1 & 2 & . & . & . & . & 10 & . & . & 13 & . & 1 \\
\hline Epipactis atrorubens & . & . & 21 & . & . & . & . & . & . & . & . & . & . & . & . & . & . & 1 & . & . & . & . & . \\
\hline Linum catharticum & . & . & 14 & . & . & . & . & . & . & . & . & . & . & . & . & . & . & . & . & . & . & . & . \\
\hline Viola odorata & . & 2 & 12 & . & . & . & 2 & . & . & 1 & . & . & . & . & . & . & . & . & . & . & . & . & . \\
\hline Ranunculus acris & . & 2 & 12 & . & . & . & 2 & . & . & . & . & . & . & . & . & . & . & . & . & . & . & . & . \\
\hline Brachythecium velutinum & . & . & 12 & . & 5 & . & 4 & . & . & . & . & . & . & 1 & . & . & . & . & . & 1 & . & . & . \\
\hline Tussilago farfara & . & . & 10 & ${ }^{\circ}$ & . & . & 2 & . & . & . & . & . & . & . & . & . & . & . & . & 1 & . & . & . \\
\hline Euphorbia cyparissias & 43 & 48 & 45 & 75 & 10 & 1 & 13 & 7 & 3 & 7 & 26 & 11 & 2 & . & . & . & . & 6 & . & 1 & . & 3 & 16 \\
\hline Arrhenatherum elatius & 2 & 9 & 28 & 72 & 4 & . & 7 & 6 & . & 10 & 56 & 12 & 4 & . & . & . & . & 3 & . & 1 & . & 1 & . \\
\hline Chaerophyllum temulum & . & . & 1 & 50 & . & . & . & . & . & . & . & 2 & . & . & . & . & . & 1 & . & . & . & . & . \\
\hline Hieracium murorum & 7 & 17 & 23 & 47 & 13 & 12 & 20 & 2 & 1 & 6 & 23 & 2 & . & . & . & . & . & 1 & . & 1 & 1 & . & 7 \\
\hline Poa pratensis & 2 & . & 26 & 38 & 7 & . & 4 & . & 1 & 6 & 5 & 10 & 5 & 1 & . & . & . & 18 & . & . & 3 & 1 & 14 \\
\hline Clinopodium vulgare & . & . & 1 & 34 & . & . & . & . & . & . & . & . & . & . & . & . & . & . & . & . & . & . & . \\
\hline Knautia arvensis & . & . & 1 & 34 & 1 & . & . & . & . & . & . & 2 & . & . & . & . & . & 4 & . & 1 & . & 1 & 2 \\
\hline Solidago canadensis & . & 2 & . & 31 & . & . & . & . & . & . & . & . & . & . & . & . & . & . & . & . & . & . & . \\
\hline Astragalus glycyphyllos & . & . & 3 & 28 & . & . & . & . & . & . & . & . & 1 & . & . & . & . & 3 & . & 1 & . & . & . \\
\hline Vicia cassubica & . & . & 4 & 25 & 1 & . & 2 & . & . & . & 12 & . & . & . & . & . & . & . & . & . & . & . & . \\
\hline Carex hirta & 3 & 2 & 1 & 22 & 2 & . & . & 4 & 3 & . & 14 & 7 & . & . & . & . & . & 1 & . & 2 & 1 & 1 & 5 \\
\hline Festuca gigantea & . & 2 & 2 & 22 & . & . & 2 & . & . & 4 & . & 5 & . & . & . & . & . & . & . & . & . & . & . \\
\hline Anthriscus sylvestris & . & . & 1 & 19 & . & . & . & . & . & . & . & 1 & . & . & . & . & . & . & . & . & . & . & . \\
\hline Trifolium repens & . & . & 3 & 16 & . & . & . & . & . & . & . & 1 & . & . & . & . & . & . & . & . & . & . & . \\
\hline Lapsana communis & . & 2 & . & 16 & . & . & . & . & . & . & . & 1 & . & 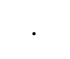 & . & . & . & . & . & . & . & . & . \\
\hline Senecio sylvaticus & 2 & 4 & 3 & . & 30 & 18 & . & 6 & 2 & 4 & . & 15 & 18 & 1 & . & . & 1 & 5 & . & 1 & 1 & 1 & . \\
\hline Teucrium scorodonia & . & . & 1 & . & 13 & 65 & 48 & . & . & . & . & 1 & . & . & . & . & . & . & . & 9 & . & . & . \\
\hline Galium saxatile & . & . & . & . & 17 & 63 & . & 2 & 3 & 8 & 2 & 17 & . & . & . & . & . & 3 & . & 14 & 1 & . & 1 \\
\hline Atrichum undulatum & . & . & . & . & 6 & 23 & 7 & . & . & 7 & . & 5 & . & . & . & . & . & . & . & 1 & . & . & 2 \\
\hline Luzula luzuloides & . & . & 4 & . & 1 & 15 & 11 & . & . & . & . & 1 & . & . & . & . & . & . & . & 2 & . & . & . \\
\hline Sambucus racemosa & . & . & . & . & 6 & 14 & 2 & . & . & 3 & . & 2 & . & . & 2 & . & . & . & . & 3 & . & . & . \\
\hline Senecio ovatus & . & . & 32 & . & 5 & 5 & 41 & . & . & . & . & . & . & . & . & . & . & . & . & 1 & . & . & . \\
\hline Melica nutans & . & 7 & 9 & 3 & 1 & . & 20 & . & . & . & . & 1 & . & . & . & . & . & . & . & 1 & . & . & 9 \\
\hline Hypnum jutlandicum & . & . & . & . & . & . & . & 87 & 1 & 1 & . & 4 & 2 & 3 & . & 2 & . & 3 & . & . & 1 & . & . \\
\hline Rhizomnium punctatum & . & . & . & . & . & . & . & 85 & 1 & . & . & 4 & . & . & . & . & . & . & . & . & . & . & . \\
\hline Brachythecium rutabulum & 18 & 41 & . & . & 5 & 5 & 2 & 67 & . & 28 & . & 6 & . & . & . & . & . & 1 & 2 & 2 & . & 2 & 1 \\
\hline Ceratodon purpureus & 2 & 9 & . & . & 1 & . & 7 & 67 & 47 & 11 & . & 6 & 35 & 10 & 5 & . & 6 & 8 & . & 2 & 1 & 3 & 2 \\
\hline Lophocolea heterophylla & . & . & . & . & . & . & . & 48 & 38 & 3 & . & 7 & . & 4 & . & . & . & 1 & . & 1 & . & 3 & 5 \\
\hline Dicranella heteromalla & . & . & . & 3 & 2 & 3 & 2 & 26 & 57 & 39 & 5 & 7 & 4 & & 1 & . & . & 3 & . & 1 & 1 & 11 & 1 \\
\hline Dicranum montanum & . & . & . & . & . & . & . & . & 15 & 1 & . & 2 & . & . & . & . & . & . & . & . & . & 1 & 1 \\
\hline Plagiothecium curvifolium & . & . & . & . & 1 & . & . & 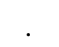 & 1 & 33 & . & 2 & & 1 & . & . & . & 1 & . & 1 & . & 2 & 1 \\
\hline
\end{tabular}


Table I. Continued.

\begin{tabular}{|c|c|c|c|c|c|c|c|c|c|c|c|c|c|c|c|c|c|c|c|c|c|c|c|}
\hline Cluster & 1 & 2 & 3 & 4 & 5 & 6 & 7 & 8 & 9 & 10 & 11 & 12 & 13 & 14 & 15 & 16 & 17 & 18 & 19 & 20 & 21 & 22 & 23 \\
\hline Number of vegetation plots & 61 & 46 & 97 & 32 & 203 & 78 & 46 & 54 & 89 & 72 & 43 & 178 & 85 & 221 & 87 & 52 & 131 & 91 & 65 & 194 & 144 & 119 & 116 \\
\hline Dryopteris dilatata & . & . & 1 & . & 7 & 30 & 11 & . & 3 & . & 81 & 30 & . & . & 3 & 8 & 1 & . & 31 & 17 & 38 & & 1 \\
\hline Oxalis acetosella & . & . & 3 & . & 3 & 23 & 39 & 2 & 7 & 1 & 72 & 40 & . & . & 1 & 6 & . & 1 & 3 & 35 & . & . & 31 \\
\hline Pteridium aquilinum & 7 & 13 & . & . & 3 & . & 26 & 9 & 12 & 28 & 54 & 9 & . & 1 & 18 & 33 & 9 & 5 & . & 40 & 33 & 21 & 36 \\
\hline Potentilla reptans & . & 2 & 1 & 19 & 1 & . & 2 & . & . & 3 & 35 & 6 & . & . & . & . & . & 1 & . & 1 & . & . & 2 \\
\hline Holcus lanatus & 2 & 2 & . & 3 & 5 & 4 & 2 & 4 & 7 & . & 26 & 17 & 6 & . & . & . & . & 1 & 5 & 1 & 8 & . & 4 \\
\hline Cladonia spec. & . & . & . & 6 & 2 & . & . & 76 & 69 & 1 & . & 8 & 91 & 84 & 1 & 18 & 29 & 11 & 2 & 2 & 25 & 27 & 3 \\
\hline Corynephorus canescens & . & . & . & . & 3 & . & . & 2 & 1 & . & . & . & 74 & 5 & . & . & 2 & 8 & . & . & . & 1 & . \\
\hline Polytrichum piliferum & . & . & . & . & . & . & . & . & 9 & 3 & . & 2 & 52 & 5 & . & . & 2 & 3 & . & 1 & 1 & . & . \\
\hline Spergula morisonii & . & . & . & . & . & . & . & . & . & . & . & . & 41 & 1 & . & . & . & . & . & . & . & . & . \\
\hline Cetraria spec. & . & . & . & . & . & . & . & . & . & . & . & . & 40 & 17 & . & . & . & . & . & . & 1 & . & . \\
\hline Cephaloziella divaricata & . & . & . & . & . & . & . & . & . & . & . & . & 31 & 15 & . & . & . & . & . & . & . & . & . \\
\hline Campylopus introflexus & & . & . & . & . & . & . & . & 4 & 3 & . & . & . & 13 & 3 & . & . & . & . & . & . & . & 1 \\
\hline Ptilidium ciliare & . & . & . & . & 2 & . & . & 19 & 19 & 1 & . & 1 & 35 & 66 & 1 & . & 17 & . & . & 2 & 3 & 39 & 3 \\
\hline Dicranum spurium & . & . & . & . & . & . & . & . & 1 & . & . & . & 11 & 29 & . & . & 8 & . & . & . & 4 & 2 & . \\
\hline Molinia caerulea & 10 & . & . & . & 4 & 1 & 15 & . & 6 & 3 & . & 7 & 1 & 4 & 97 & 40 & 8 & 1 & 52 & 59 & 77 & 4 & 3 \\
\hline Ledum palustre & . & . & . & . & . & . & . & . & . & . & . & . & . & . & 53 & . & 1 & . & 49 & 1 & . & . & . \\
\hline Picea abies & . & . & . & 6 & 2 & 1 & . & 7 & 11 & 1 & . & 17 & . & . & 47 & 4 & 5 & . & 3 & 21 & 12 & 15 & 25 \\
\hline Potentilla erecta & . & . & . & . & 3 & 10 & 22 & . & . & 1 & . & 7 & . & . & 26 & . & . & 1 & 2 & 6 & 15 & . & 10 \\
\hline Vaccinium uliginosum & . & . & . & . & . & . & . & . & . & . & . & . & . & . & 21 & . & 1 & . & 12 & . & . & . & . \\
\hline Erica tetralix & . & . & . & . & . & . & . & . & . & . & . & . & . & . & 18 & . & 1 & . & 2 & . & 1 & . & . \\
\hline Sphagnum capillifolium & . & . & . & . & . & . & . & . & . & . & . & . & . & . & 14 & . & . & . & . & . & . & . & . \\
\hline Salix repens & . & . & . & . & . & . & . & . & . & . & . & . & . & . & 14 & . & . & 3 & . & . & 3 & . & . \\
\hline Empetrum nigrum & . & . & . & . & . & . & . & . & . & . & . & . & . & . & . & 75 & . & 8 & . & . & . & 2 & . \\
\hline Juniperus communis & 3 & 13 & . & 34 & 1 & . & . & . & 7 & 1 & 23 & 4 & 2 & 2 & 2 & 65 & . & 18 & . & 2 & . & 19 & 37 \\
\hline Trientalis europaea & . & . & . & . & . & . & . & . & 2 & . & 2 & 1 & . & . & 8 & 60 & . & 8 & . & 18 & . & . & 3 \\
\hline Lonicera periclymenum & 2 & 9 & 2 & 6 & 2 & . & 2 & 4 & 18 & . & . & 21 & . & . & . & 25 & . & 6 & . & 18 & 2 & 1 & 2 \\
\hline Ilex aquifolium & . & . & . & . & . & . & . & . & . & . & . & . & . & . & . & 12 & . & . & . & 1 & . & . & . \\
\hline Genista pilosa & 7 & 4 & 2 & . & 3 & 1 & . & . & . & . & . & . & . & 2 & . & . & 12 & . & . & . & 2 & 4 & 6 \\
\hline Carex arenaria & 36 & . & . & 3 & 6 & . & . & 19 & 27 & 1 & . & 8 & 24 & 6 & . & . & . & 43 & & 9 & 1 & 13 & 2 \\
\hline Hieracium umbellatum & 5 & 9 & 1 & . & 1 & . & . & . & 1 & 3 & . & 1 & 19 & 5 & . & . & . & 30 & & 1 & . & 3 & 1 \\
\hline Polypodium vulgare & . & . & . & . & 3 & . & . & . & . & . & 2 & . & . & . & . & . & . & 16 & & . & . & 1 & . \\
\hline Goodyera repens & . & . & . & . & . & . & . & . & . & . & . & . & . & . & . & 4 & . & 18 & & 2 & . & . & . \\
\hline Moneses uniflora & . & . & 4 & . & 8 & . & . & . & . & . & . & . & . & . & . & 8 & . & 18 & & 2 & . & 2 & 1 \\
\hline Pyrola chlorantha & . & . & 1 & 9 & 1 & . & . & . & . & . & . & . & 1 & . & . & 6 & . & 13 & & . & . & 2 & . \\
\hline Polypodium interjectum & . & . & . & . & . & . & . & . & . & . & . & . & 1 & 1 & . & . & . & 14 & & . & . & . & 3 \\
\hline Eriophorum vaginatum & . & . & . & . & . & . & . & . & . & . & . & . & . & . & 18 & . & . & . & 79 & 1 & . & . & . \\
\hline Vaccinium oxycoccus & . & . & . & . & . & . & . & . & . & . & . & . & . & . & 12 & . & . & . & 75 & . & . & . & . \\
\hline Sphagnum fallax & . & . & . & . & . & . & . & . & . & . & . & . & . & . & 26 & . & . & . & 52 & 1 & . & . & . \\
\hline Aulacomnium palustre & . & . & . & . & . & . & . & . & . & . & . & 1 & 1 & . & 3 & . & . & . & 51 & 1 & 1 & . & . \\
\hline Sphagnum palustre & . & . & . & & . & . & . & 2 & 1 & . & . & 1 & . & . & 22 & . & 1 & . & 46 & 6 & . & . & . \\
\hline Polytrichum strictum & . & . & . & . & . & . & . & . & . & . & . & . & . & . & . & . & . & . & 46 & . & . & . & . \\
\hline Sphagnum angustifolium & . & . & . & . & . & . & . & . & . & . & . & . & . & . & . & . & . & . & 37 & . & . & . & . \\
\hline
\end{tabular}


Table I. Continued.

\begin{tabular}{|c|c|c|c|c|c|c|c|c|c|c|c|c|c|c|c|c|c|c|c|c|c|c|c|}
\hline Cluster & 1 & 2 & 3 & 4 & 5 & 6 & 7 & 8 & 9 & 10 & 11 & 12 & 13 & 14 & 15 & 16 & 17 & 18 & 19 & 20 & 21 & 22 & 23 \\
\hline Number of vegetation plots & 61 & 46 & 97 & 32 & 203 & 78 & 46 & 54 & 89 & 72 & 43 & 178 & 85 & 221 & 87 & 52 & 131 & 91 & 65 & 194 & 144 & 119 & 116 \\
\hline$\overline{P o l y t r i c h u m ~ c o m m u n e ~}$ & & . & . & . & . & . & . & . & . & . & . & . & . & . & 30 & . & 1 & . & 34 & 3 & 2 & 1 & \\
\hline Eriophorum angustifolium & & . & . & . & . & . & . & . & . & . & . & . & . & . & 3 & . & . & . & 32 & 1 & 1 & . & 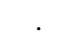 \\
\hline Peucedanum palustre & . & . & . & . & . & . & . & . & . & . & . & 1 & . & . & . & . & . & . & 29 & 1 & 1 & . & . \\
\hline Sphagnum magellanicum & . & . & . & . & . & . & . & . & . & . & . & . & . & . & . & . & . & . & 29 & . & . & . & . \\
\hline Andromeda polifolia & . & . & . & . & . & . & . & . & . & . & . & . & . & . & 7 & . & . & . & 23 & . & . & . & . \\
\hline Tetraphis pellucida & . & . & . & . & . & . & . & . & . & . & . & . & . & . & . & . & . & . & 23 & . & . & . & . \\
\hline Hydrocotyle vulgaris & . & . & . & . & . & . & . & . & . & . & . & 3 & . & . & 1 & . & . & . & 22 & 1 & 9 & . & . \\
\hline Lysimachia thyrsiflora & . & . & . & . & . & . & . & . & . & . & . & . & . & . & . & . & . & . & 20 & 1 & 1 & . & . \\
\hline Potentilla palustris & . & . & . & . & . & . & . & . & . & . & . & 1 & . & . & . & . & . & . & 20 & . & . & . & . \\
\hline Alnus glutinosa & . & . & . & . & . & . & 2 & 2 & . & . & . & 3 & . & 1 & 2 & . & . & 3 & 17 & 6 & 1 & . & . \\
\hline Agrostis canina agg. & . & . & . & . & . & . & . & . & . & . & . & . & . & . & 3 & . & . & . & 17 & . & . & . & . \\
\hline Drosera rotundifolia & . & . & . & . & . & . & . & . & . & . & . & . & . & . & . & . & . & . & 17 & . & . & . & . \\
\hline Carex lasiocarpa & . & . & . & . & . & . & . & . & . & . & . & . & . & . & . & . & . & . & 15 & . & . & . & . \\
\hline Carex canescens & . & . & . & . & . & . & . & . & . & . & . & 1 & . & . & 1 & . & . & . & 14 & 1 & . & . & . \\
\hline Cephalozia connivens & . & . & . & . & . & . & . & . & . & . & . & . & . & . & . & . & . & . & 14 & . & . & . & . \\
\hline Juncus effusus & . & . & . & . & 2 & . & . & . & 1 & 4 & 2 & 3 & . & . & . & . & . & . & 12 & 2 & . & 2 & 2 \\
\hline Dicranella cerviculata & . & . & . & . & . & . & . & . & . & . & . & . & . & . & 2 & . & . & . & 11 & 1 & . & . & . \\
\hline Calliergon stramineum & . & . & . & . & . & . & . & . & . & . & . & . & . & . & . & . & . & . & 11 & . & . & . & \\
\hline Leucobryum glaucum & & . & . & . & 7 & . & . & . & 20 & 1 & 5 & 1 & 2 & 41 & 25 & 25 & 36 & 11 & 14 & 24 & 57 & 22 & 17 \\
\hline Fagus sylvatica & 3 & 22 & 4 & 9 & 12 & 58 & 48 & 37 & 44 & 25 & 44 & 44 & 1 & 1 & . & 65 & 2 & 14 & 9 & 41 & 6 & 44 & 92 \\
\hline Luzula pilosa & 7 & 15 & 2 & 19 & 25 & 68 & 33 & 15 & 38 & 19 & 58 & 25 & . & 1 & . & 23 & . & 15 & 2 & 37 & 37 & 19 & 81 \\
\hline \multicolumn{24}{|l|}{ Cluster group 1-12 } \\
\hline Rubus idaeus & 20 & 11 & 84 & 69 & 51 & 86 & 98 & 28 & 26 & 25 & 95 & 73 & . & . & . & . & . & 5 & 2 & 36 & 4 & 2 & 21 \\
\hline Calamagrostis epigejos & 76 & 70 & 95 & 44 & 75 & 92 & 96 & 4 & 12 & 28 & 30 & 48 & 20 & 6 & . & . & 1 & 24 & . & 28 & 38 & 23 & 12 \\
\hline Agrostis capillaris & 84 & 70 & 54 & 47 & 71 & 54 & 48 & 22 & 14 & 57 & 74 & 52 & 27 & 9 & . & . & 5 & 11 & . & 16 & 13 & 17 & 7 \\
\hline Rubus fruticosus agg. & 18 & 4 & 93 & 16 & 34 & 64 & 91 & 15 & 17 & 24 & 42 & 28 & 1 & . & 7 & 2 & . & 3 & . & 39 & 13 & 3 & 5 \\
\hline Moehringia trinervia & 23 & 94 & 31 & 50 & 36 & 22 & 30 & 9 & 14 & 72 & 30 & 58 & 1 & . & . & . & . & 1 & 2 & 9 & 15 & 1 & 9 \\
\hline Brachypodium sylvaticum & . & 46 & 99 & 97 & 3 & 3 & 96 & . & . & 4 & 14 & 5 & . & . & . & . & . & 4 & . & 2 & . & 1 & 14 \\
\hline Prunus serotina & 57 & 96 & 6 & 59 & 19 & 19 & . & 57 & 32 & 93 & . & 33 & 8 & 3 & . & . & . & 3 & . & 3 & 4 & 17 & 1 \\
\hline Epilobium angustifolium & . & 39 & 51 & 28 & 46 & 78 & 61 & 2 & 2 & 14 & 12 & 27 & 6 & 1 & 9 & . & . & . & 2 & 8 & 15 & 4 & 4 \\
\hline Mycelis muralis & 7 & 89 & 69 & 81 & 45 & 31 & 78 & 2 & 1 & 13 & 7 & 22 & . & . & . & . & . & . & . & 3 & 8 & 1 & 3 \\
\hline Rumex acetosella & 48 & 65 & 3 & 22 & 67 & 46 & 22 & 43 & 35 & 40 & 77 & 52 & 61 & 13 & . & . & 6 & 19 & . & 11 & 19 & 16 & 24 \\
\hline Viola reichenbach. et rivin. & 3 & 59 & 65 & 41 & 15 & 44 & 87 & . & 1 & 13 & 67 & 5 & . & . & . & . & . & 1 & . & 5 & 3 & 1 & 20 \\
\hline Galium mollugo agg. & 2 & 2 & 20 & 25 & 1 & 3 & 2 & 6 & 1 & 1 & 23 & 7 & . & . & . & . & . & 23 & . & . & . & . & 6 \\
\hline Fragaria vesca & 7 & 89 & 100 & 97 & 5 & 4 & 85 & . & . & 1 & 26 & 3 & . & . & . & . & . & 1 & . & 1 & 1 & 2 & 10 \\
\hline Hieracium laevigatum & 61 & 50 & 46 & 25 & 34 & 28 & 24 & . & . & 13 & 12 & 4 & 2 & 2 & . & . & . & . & . & 1 & 14 & 4 & 1 \\
\hline Impatiens parviflora & 5 & 28 & 1 & 31 & 3 & . & 2 & . & 2 & 40 & . & 11 & . & . & . & . & . & 3 & . & . & . & . & . \\
\hline Galeopsis tetrahit et bifida & $\cdot$ & 4 & 6 & 6 & 31 & 23 & 48 & 2 & 6 & 18 & 23 & 26 & 17 & . & . & . & . & . & . & 6 & 2 & . & . \\
\hline Taraxacum officinale agg. & 44 & 67 & 47 & 44 & 20 & 1 & 9 & . & 3 & 6 & . & 5 & 4 & 1 & . & . & . & 6 & . & 1 & 2 & 1 & 1 \\
\hline Ribes uva-crispa & 2 & 9 & 14 & 3 & . & 1 & 11 & . & . & 6 & 16 & 3 & . & . & . & . & . & . & . & . & . & . & . \\
\hline Urtica dioica & . & 11 & . & 31 & . & . & 2 & . & 3 & 17 & 17 & 19 & . & . & . & . & . & 3 & 3 & 1 & . & . & 6 \\
\hline Linaria vulgaris & 31 & 26 & 17 & 9 & 5 & . & 4 & . & . & 1 & 2 & 2 & 2 & . & . & . & . & 1 & . & . & . & . & . \\
\hline Sambucus nigra & 7 & 11 & 9 & 3 & 3 & 1 & 2 & 2 & . & 13 & 9 & 12 & 2 & . & . & . & . & . & . & . & . & . & . \\
\hline Poa nemoralis & 5 & 7 & 21 & 28 & 3 & . & 7 & . & . & 28 & 5 & 6 & . & . & . & . & . & . & . & 1 & 1 & 1 & 2 \\
\hline Epilobium montanum & & 13 & 27 & 28 & 1 & 1 & 22 & . & . & . & . & 2 & . & . & . & . & . & . & . & . & . & . & . \\
\hline
\end{tabular}


Table I. Continued.

\begin{tabular}{|c|c|c|c|c|c|c|c|c|c|c|c|c|c|c|c|c|c|c|c|c|c|c|c|}
\hline Cluster & 1 & 2 & 3 & 4 & 5 & 6 & 7 & 8 & 9 & 10 & 11 & 12 & 13 & 14 & 15 & 16 & 17 & 18 & 19 & 20 & 21 & 22 & 23 \\
\hline Number of vegetation plots & 61 & 46 & 97 & 32 & 203 & 78 & 46 & 54 & 89 & 72 & 43 & 178 & 85 & 221 & 87 & 52 & 131 & 91 & 65 & 194 & 144 & 119 & 116 \\
\hline Dactylis glomerata et polyg. & 3 & 4 & 20 & 44 & 2 & . & 4 & $\cdot$ & 1 & 8 & 49 & 9 & . & . & . & . & . & 13 & . & 1 & $\cdot$ & & 3 \\
\hline Dryopteris filix-mas & . & 11 & 8 & 6 & 2 & 17 & 26 & $\cdot$ & 1 & 1 & 26 & 21 & . & . & . & . & . & 1 & $\cdot$ & 2 & 1 & 1 & 3 \\
\hline Viburnum opulus & 5 & 11 & 22 & 28 & 1 & . & 7 & . & . & . & . & 2 & . & . & . & . & . & . & . & $\cdot$ & . & . & . \\
\hline Robinia pseudoacacia & 3 & 15 & 2 & 6 & 2 & 1 & . & . & . & 15 & . & 1 & 5 & 2 & . & . & . & . & . & . & . & 4 & \\
\hline \multicolumn{24}{|l|}{ Cluster group 13-23 } \\
\hline Calluna vulgaris & 38 & 9 & 24 & 25 & 47 & 21 & 4 & 6 & 11 & 1 & 16 & 6 & 22 & 61 & 53 & 77 & 89 & 21 & 17 & 13 & 51 & 67 & 45 \\
\hline Vaccinium vitis-idaea & 2 & 44 & 1 & 13 & 8 & . & . & . & 6 & . & 7 & 1 & . & 18 & 83 & 94 & 95 & 1 & 3 & 16 & 39 & 9 & 2 \\
\hline Dicranum scoparium & 5 & . & . & 6 & 17 & 22 & 9 & 2 & 42 & 11 & 26 & 12 & 74 & 84 & 1 & 29 & 31 & 14 & 14 & 6 & 27 & 81 & 8 \\
\hline Dicranum polysetum & . & . & . & . & 12 & . & . & 32 & 78 & 4 & . & 22 & 12 & 43 & 8 & 49 & 28 & 14 & . & 13 & 35 & 75 & 77 \\
\hline Pohlia nutans & 2 & . & . & 9 & 5 & . & . & . & 5 & 71 & . & 10 & 68 & 65 & 2 & . & 35 & 8 & 26 & 3 & 47 & 51 & 6 \\
\hline Polytrichum juniperinum & . & . & . & . & 1 & . & . & . & 1 & . & . & & 4 & 3 & 3 & . & 11 & 3 & 2 & 1 & 1 & 6 & 3 \\
\hline
\end{tabular}

Other trees and shrubs

Quercus robur et petraea

Sorbus aucuparia

Betula pendula et pubesc.

Pinus sylvestris

Frangula alnus

Quercus rubra

Euonymus europaea

Populus tremula

Corylus avellana

Carpinus betulus

Berberis vulgaris

Cornus sanguinea

Crataegus laevigata

Rosa spec.

$$
\begin{aligned}
& \begin{array}{lllllllllllllllllllllll}
93 & 98 & 83 & 81 & 88 & 78 & 80 & 91 & 99 & 89 & 95 & 89 & 69 & 57 & 28 & 46 & 54 & 50 & 8 & 68 & 79 & 88 & 84
\end{array} \\
& \begin{array}{lllllllllllllllllllllll}
69 & 87 & 87 & 59 & 79 & 94 & 85 & 82 & 90 & 90 & 86 & 84 & 12 & 8 & 22 & 40 & 2 & 34 & 2 & 67 & 58 & 48 & 43
\end{array} \\
& \begin{array}{lllllllllllllllllllllll}
69 & 57 & 62 & 22 & 54 & 73 & 44 & 59 & 74 & 75 & 79 & 65 & 44 & 27 & 47 & 28 & 18 & 21 & 79 & 68 & 86 & 53 & 52
\end{array} \\
& \begin{array}{llllllllllllllllllllllllll}
38 & 54 & 24 & 19 & 75 & 41 & 22 & 48 & 65 & 60 & 33 & 24 & 66 & 52 & 24 & 49 & 47 & 19 & 8 & 21 & 56 & 72 & 38
\end{array} \\
& \begin{array}{llllllllllllllllllllllllll}
8 & 13 & 66 & 34 & 65 & 47 & 50 & 50 & 66 & 17 & 47 & 59 & 17 & 3 & 12 & 2 & 1 & 9 & 20 & 54 & 72 & 21 & 22
\end{array} \\
& \begin{array}{lllllllllllllllllllllll}
. & 20 & 5 & 9 & 6 & 36 & 9 & 22 & 19 & 29 & . & 3 & 1 & 8 & . & . & 2 & 1 & . & 5 & . & 13 & .
\end{array}
\end{aligned}
$$

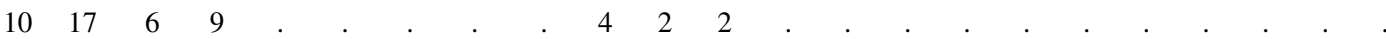

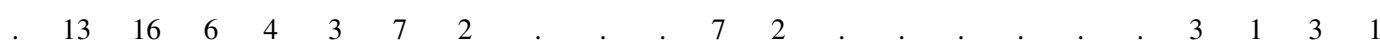

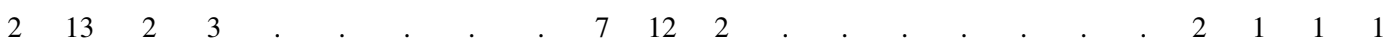

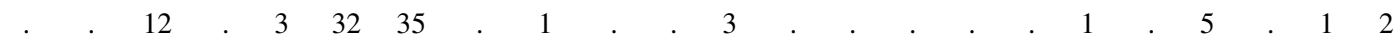

$$
\begin{aligned}
& \begin{array}{llll}
3 & 7 & 11 & 19
\end{array} \\
& \begin{array}{llllll}
. & 4 & 13 & 22 & . & 2
\end{array}
\end{aligned}
$$

Other dwarf shrubs.herbs and bryophytes

\begin{tabular}{lcccccccccccccccccccccccc} 
Deschampsia flexuosa & 75 & 100 & 100 & 78 & 99 & 100 & 98 & 100 & 100 & 85 & 93 & 91 & 79 & 82 & 24 & 96 & 41 & 76 & 31 & 85 & 90 & 97 & 78 \\
Pleurozium schreberi & 49 & 70 & 24 & 53 & 68 & 53 & 9 & 50 & 93 & 28 & 95 & 56 & 17 & 67 & 67 & 98 & 97 & 29 & 31 & 54 & 72 & 98 & 98 \\
Vaccinium myrtillus & 3 & 20 & 49 & 44 & 67 & 99 & 94 &. & 63 & 10 & 21 & 27 & 2 & 28 & 92 & 92 & 94 & 14 & 34 & 88 & 92 & 71 & 98 \\
Hypnum cupressiforme & 10 & 9 & 20 & 6 & 67 & 72 & 33 & 2 & 93 & 29 & 7 & 32 & 38 & 81 & 33 &. & 73 & 11 & 3 & 26 & 49 & 84 & 4 \\
Scleropodium purum & 18 & 91 & 61 & 97 & 39 & 78 & 26 & 98 & 74 & 40 & 54 & 60 & 4 & 1 & 2 & 35 & 4 & 38 & 8 & 35 & 36 & 35 & 57 \\
Carex pilulifera & 25 & 30 & 12 & 6 & 64 & 78 & 15 & 6 & 28 & 49 & 16 & 21 & 4 & 11 & 12 &. & 32 & 1 &. & 28 & 69 & 15 & 24 \\
Dryopteris carthusiana & 2 & 13 & 8 & 3 & 22 & 67 & 44 & 78 & 81 & 29 &. & 74 &. & 1 & 14 &. & 2 & 13 & 5 & 43 & 1 & 11 & 42 \\
Melampyrum pratense & 95 & 78 & 10 & 72 & 27 & 15 & 13 & 2 & 23 & 15 & 9 & 13 &. & 3 & 7 & 73 & 41 & 16 &. & 11 & 42 & 37 & 48 \\
Anthoxanthum odoratum & 18 & 59 & 6 & 41 & 37 & 6 & 7 & 11 & 9 & 15 & 49 & 24 & 13 & 1 & 1 & 10 & 2 & 31 & 2 & 8 & 17 & 9 & 37 \\
Festuca ovina agg. & 98 & 80 & 53 & 69 & 77 & 18 & 13 &. & 2 & 15 &. & 11 & 59 & 35 &. &. & 25 & 18 &. & 5 & 1 & 23 & 40 \\
Danthonia decumbens & 44 & 39 & 8 & 3 & 23 & 10 & 4 &. &. & 4 & 16 & 4 &. & 1 & 5 &. & 5 &. &. & 1 & 6 & 13 & 10 \\
Veronica officinalis & 18 & 72 & 56 & 31 & 31 & 12 & 20 &. &. & 7 & 5 & 10 & 2 &. &. &. &. & 18 &. & 1 & 1 & 5 & 9 \\
Polytrichum formosum & 2 & 4 &. & 3 & 8 &. &. & 24 & 35 & 24 & 14 & 19 &. & 5 & 16 &. & 5 & 3 & 9 & 20 & 20 & 11 & 22 \\
Maianthemum bifolium & 3 &. & 7 &. & 2 & 12 & 41 &. & 7 & 8 & 9 & 7 &. &. & 1 & 27 & 1 & 3 &. & 22 & 24 & 1 & 4 \\
Luzula multiflora et camp. & 7 & 13 & 3 & 6 & 17 & 10 & 7 &. & 2 & 7 &. & 10 & 6 & 5 & 1 & 2 & 18 & 10 &. & 1 & 19 & 26 & 15 \\
Viola canina & 36 & 46 & 23 & 53 & 6 & 1 & 11 &. &. &. &. & 4 & 2 & 1 &. &. &. & 4 &. &. &. & 3 &. \\
Achillea millefolium agg. & 10 & 7 & 34 & 31 & 5 &. &. &. &. & 1 & 5 & 1 & 1 &. &. &. &. & 10 &. &. &. & 1 & 2 \\
Orthilia secunda & 2 & 39 & 18 & 38 & 4 & 3 &. &. & 1 & 1 &. &. & 2 &. &. & 4 & 1 & 16 &. & 1 & 4 &. & 2 \\
\hline
\end{tabular}


Table I. Continued.

\begin{tabular}{|c|c|c|c|c|c|c|c|c|c|c|c|c|c|c|c|c|c|c|c|c|c|c|c|}
\hline Cluster & 1 & 2 & 3 & 4 & 5 & 6 & 7 & 8 & 9 & 10 & 11 & 12 & 13 & 14 & 15 & 16 & 17 & 18 & 19 & 20 & 21 & 22 & 23 \\
\hline Number of vegetation plots & 61 & 46 & 97 & 32 & 203 & 78 & 46 & 54 & 89 & 72 & 43 & 178 & 85 & 221 & 87 & 52 & 131 & 91 & 65 & 194 & 144 & 119 & 116 \\
\hline Holcus mollis & 16 & 2 & 10 & 6 & 8 & 3 & 11 & . & 3 & 15 & 16 & 19 & 2 & 1 & . & . & 1 & 4 & & 8 & 19 & 3 & . \\
\hline Calamagrostis arundinacea & . & 2 & 4 & 3 & 2 & 6 & 13 & . & . & 17 & . & 5 & . & . & . & . & . & . & 20 & 12 & 4 & 3 & 7 \\
\hline Veronica chamaedrys & 3 & 65 & 28 & 72 & 3 & 1 & 7 & 2 & 1 & 6 & 2 & 3 & . & . & . & . & . & 7 & . & . & 1 & . & 6 \\
\hline Hylocomium splendens & . & 2 & . & 16 & 1 & . & . & . & 2 & . & . & 2 & . & . & 7 & 8 & 5 & 9 & 2 & 5 & . & 10 & 21 \\
\hline Galium verum & 13 & 26 & 18 & 34 & 9 & . & 2 & . & 1 & 3 & 12 & 4 & 8 & . & . & . & . & 9 & . & 2 & . & 2 & . \\
\hline Polygonatum odoratum & 71 & 78 & 1 & 19 & 6 & 1 & 2 & . & . & 7 & . & 3 & . & . & . & . & . & . & . & 1 & . & . & 1 \\
\hline Solidago virgaurea & 54 & 50 & 3 & 22 & 3 & . & . & . & . & . & . & 2 & 1 & 1 & . & . & . & 4 & . & 1 & . & 1 & . \\
\hline Cirsium palustre & . & . & 33 & 13 & 1 & . & 26 & . & . & . & . & 2 & . & . & 1 & . & . & . & 2 & 1 & 2 & . & . \\
\hline Deschampsia cespitosa & . & . & 9 & 3 & 1 & . & 13 & 2 & 1 & 4 & . & 17 & . & . & . & . & . & 1 & 2 & 3 & 6 & . & 4 \\
\hline Rumex acetosa & 3 & 13 & 16 & 6 & 10 & . & 4 & . & . & 4 & 14 & 4 & 4 & . & . & . & . & 1 & 2 & . & 4 & . & . \\
\hline Hieracium sabaudum & 13 & 13 & 7 & 6 & 3 & . & 7 & . & . & . & . & 5 & 1 & . & . & . & . & . & . & . & . & . & . \\
\hline Scorzonera humilis & 15 & 11 & . & . & 1 & . & . & . & . & . & . & . & . & 1 & . & 4 & . & 1 & . & 1 & . & . & 2 \\
\hline Nardus stricta & . & . & . & . & 3 & 3 & . & . & . & . & . & . & . & 1 & 20 & . & 6 & . & . & 1 & 10 & 1 & . \\
\hline Solanum dulcamara & . & 2 & 12 & 3 & . & . & . & . & . & 1 & . & 1 & 1 & . & . & . & . & 1 & 9 & 1 & . & . & . \\
\hline Epipactis helleborine & 18 & . & 19 & 28 & . & . & . & . & . & . & . & . & . & . & . & . & . & . & . & . & 1 & . & . \\
\hline Hypochaeris radicata & 34 & 28 & 1 & . & 17 & 1 & . & 7 & 3 & 3 & . & 1 & 15 & 3 & 1 & 10 & 1 & 11 & . & . & 6 & 1 & . \\
\hline Cerastium arvense & 12 & 4 & . & . & . & . & . & . & . & . & . & 1 & . & . & . & . & 1 & 1 & . & . & . & . & . \\
\hline Eurhynchium striatum & . & . & 17 & . & 9 & 15 & 13 & . & . & . & . & . & . & . & . & . & . & . & . & 1 & . & . & . \\
\hline Brachythecium spec. & . & . & . & 28 & 2 & . & . & . & 3 & 1 & . & 10 & 5 & . & . & . & . & . & . & . & 1 & 9 & . \\
\hline Plantago lanceolata & 5 & 7 & 13 & 22 & 1 & . & . & . & . & . & . & . & 4 & . & . & . & . & 4 & . & . & . & . & . \\
\hline Lathyrus linifolius & . & 15 & 3 & 22 & 1 & . & 11 & . & . & . & . & . & . & . & . & . & . & . & . & . & . & . & 11 \\
\hline Mnium hornum & . & . & . & . & 4 & 10 & 13 & . & 2 & 4 & 7 & . & . & . & . & . & . & . & . & 5 & . & . & 1 \\
\hline Lophocolea bidentata & . & 7 & . & . & 1 & . & . & . & 2 & 1 & . & 2 & . & 2 & . & 2 & . & . & 3 & 7 & . & 1 & 3 \\
\hline Galium rotundifolium & . & . & 2 & . & 6 & 14 & 11 & . & . & . & . & 1 & . & . & . & . & . & . & . & . & . & . & . \\
\hline Anthericum ramosum & . & 11 & 3 & 9 & 2 & . & . & . & . & 1 & 5 & . & 2 & 1 & . & . & . & 3 & . & . & . & 1 & . \\
\hline Galium aparine & . & 22 & 19 & 25 & . & 1 & 2 & . & . & 1 & . & 9 & . & . & . & . & . & 4 & . & 1 & . & . & . \\
\hline Eupatorium cannabinum & . & . & 11 & . & . & . & 7 & . & . & . & . & . & . & . & . & . & . & . & . & 1 & . & . & . \\
\hline Milium effusum & . & . & . & . & . & . & 7 & . & . & . & 7 & 11 & . & . & . & . & . & . & . & 3 & . & . & . \\
\hline Thymus serpyllum & 13 & . & . & 13 & . & . & . & . & . & . & . & . & 7 & 1 & . & . & 2 & 3 & . & . & . & 3 & . \\
\hline Galium boreale & 2 & 15 & 1 & 13 & . & . & . & . & . & 1 & . & . & . & . & . & . & . & . & . & . & . & . & . \\
\hline Ajuga genevensis & 2 & 15 & 22 & 13 & 2 & . & 2 & . & . & . & . & . & . & . & . & . & . & 4 & . & . & . & . & . \\
\hline Lysimachia vulgaris & 2 & . & . & . & . & . & 4 & . & . & . & . & 5 & . & . & . & . & . & . & 12 & 10 & 21 & . & . \\
\hline Geranium robertianum & . & 39 & 33 & 41 & . & . & 9 & . & . & 10 & . & 4 & . & . & . & . & . & . & . & . & . & . & 1 \\
\hline Torilis japonica & . & 54 & 45 & 16 & . & . & 2 & . & . & 1 & . & 1 & . & . & . & . & . & . & . & . & . & . & . \\
\hline Geum urbanum & . & 28 & 30 & 38 & . & . & 2 & . & . & 4 & . & 1 & . & . & . & . & . & . & . & . & . & . & . \\
\hline Agrimonia eupatoria & . & 57 & 29 & 53 & . & . & 7 & . & . & . & . & . & 1 & . & . & . & . & 6 & . & . & . & . & . \\
\hline Cirsium vulgare & . & . & 32 & 28 & 1 & 1 & . & . & . & . & . & 1 & . & . & . & . & . & . & . & . & . & . & . \\
\hline Poa angustifolia & 41 & 57 & . & . & . & . & . & . & . & 1 & . & . & 4 & . & . & . & . & . & . & . & . & . & . \\
\hline Alliaria petiolata & . & 9 & . & 13 & . & . & . & . & . & 3 & . & 1 & . & . & . & . & . & . & . & . & . & . & . \\
\hline Daucus carota & . & . & 14 & 22 & 1 & . & . & . & . & . & . & . & . & . & . & . & . & 3 & . & . & . & . & . \\
\hline Lathyrus pratensis & . & . & 12 & 13 & . & . & . & . & . & . & . & . & . & . & . & . & . & 3 & . & . & . & . & . \\
\hline Plagiomnium undulatum & . & 7 & 10 & . & . & . & . & . & . & . & . & 1 & . & . & . & . & . & . & . & . & . & . & . \\
\hline Cardaminopsis arenosa & 5 & 11 & 4 & . & . & . & . & . & . & . & . & 1 & . & . & . & . & 2 & 1 & . & . & . & . & 1 \\
\hline Vicia tetrasperma & . & 26 & 25 & 31 & . & . & 4 & . & . & . & . & . & . & . & . & . & . & . & . & . & . & . & . \\
\hline Prunella vulgaris & . & . & 11 & 22 & . & . & . & . & . & . & . & . & . & . & . & - & . & 4 & . & . & . & . & . \\
\hline Placynthiella icmalea & . & . & . & . & . & . & . & . & . & . & . & . & 9 & 11 & . & & . & 1 & . & . & . & . & . \\
\hline
\end{tabular}



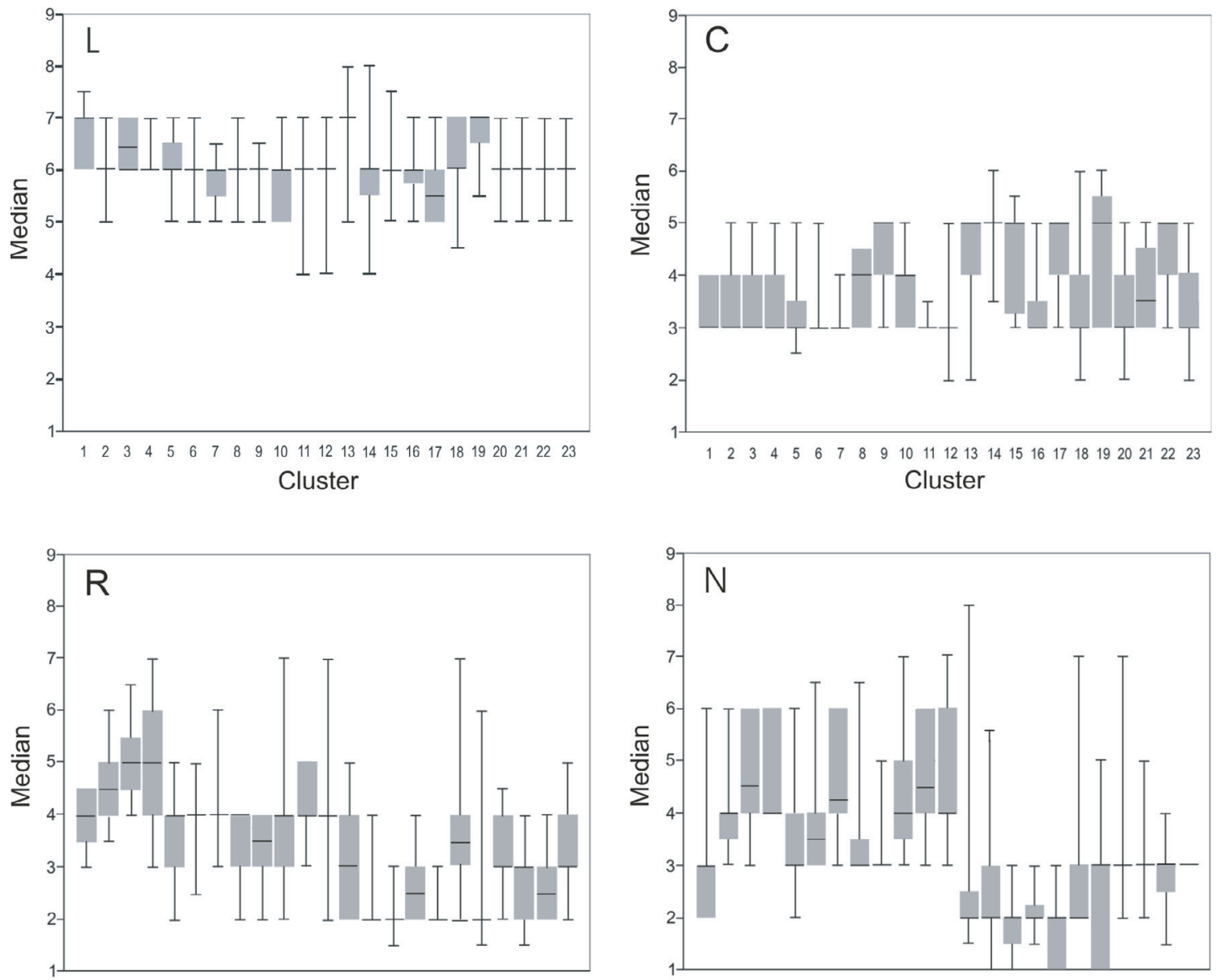

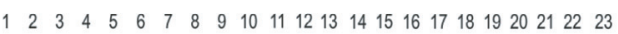
Cluster

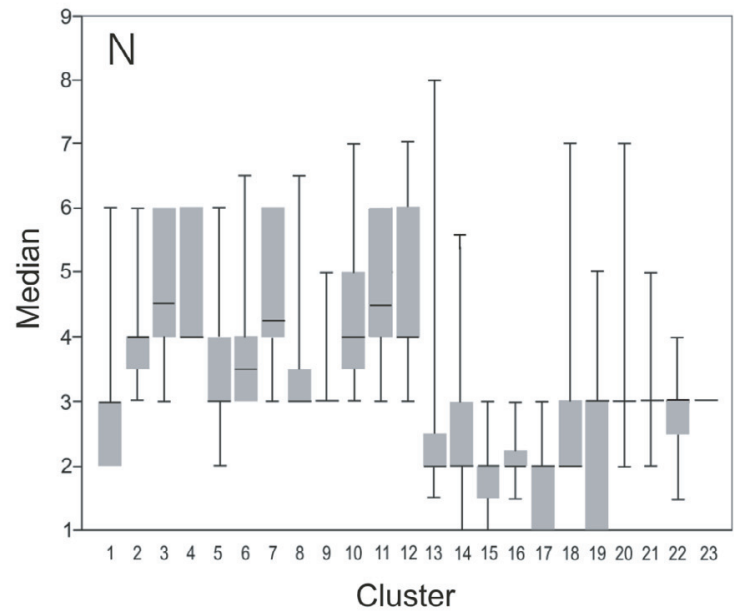

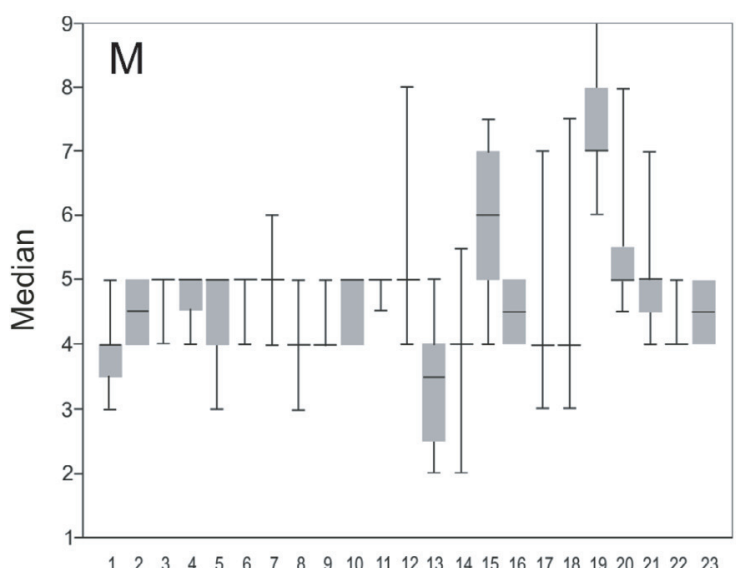

Cluster
Figure 2. Medians of Ellenberg indicator values for light $(\mathrm{L})$, continentality $(\mathrm{C})$, soil reaction $(\mathrm{R})$, nitrogen $(\mathrm{N})$, and moisture (M) given for all clusters (1-23); medians presented as Box-andWhisker-plots with quartiles, minima, and maxima given. groups. For example, the medians $(\leq 4)$ for soil reaction of the communities 1 and 5-12 are highly significantly (MannWhitney test, $p<0.01$ ) lower compared with the medians of the communities $2-4$. As indicated by these medians, the latter communities grow on moderately acid to nearly neutral sites.
The ecological difference between the two main cluster groups is also indicated by the medians of the nitrogen values $(\mathrm{N})$, which is even more distinct than the values for soil reaction (Fig. 2). Almost all medians of this indicator value are highly significantly $(p<0.01)$ higher in the cluster group $1-12$ than 
Table II. Non-indigenous species recorded in NE German pine forests with information on their life form, origin, Ellenberg ecological indicator values, and establishment in natural habitats in Central Europe. References for the data: [16] (F), [15] (E), [32] (K), [40] (L), [4] (B), [48] (O).

\begin{tabular}{|c|c|c|c|c|c|c|c|c|}
\hline \multirow[t]{2}{*}{ Non-indigenous species } & \multirow[t]{2}{*}{ Growth form } & \multirow[t]{2}{*}{ Origin } & \multicolumn{5}{|c|}{ Ellenberg indicator values } & \multirow[t]{2}{*}{ Established in natural habitats in Central Europe } \\
\hline & & & $\mathrm{L}$ & $\mathrm{C}$ & $\mathrm{M}$ & $\mathrm{R}$ & $\mathrm{N}$ & \\
\hline Acer negundo & Tree & N America & (5) & 6 & 6 & 7 & 7 & Floodplain forests \\
\hline Amelanchier alnifolia & Shrub, tree & N America & - & - & - & - & - & - \\
\hline Campylopus introflexus & Bryophyte & S hemisphere & 8 & 3 & 2 & 2 & - & - \\
\hline Conyza canadensis & Annual herb & $\mathrm{N}$ America & 8 & $\mathrm{x}$ & 4 & $\mathrm{x}$ & 5 & Pioneer communities in floodplains and at seashores \\
\hline Impatiens parviflora & Annual herb & E Asia & 4 & 5 & 5 & $\mathrm{x}$ & 6 & Floodplain and beech forests \\
\hline Ligustrum vulgare & Shrub & S Europe, Asia & 7 & 3 & 4 & 8 & 3 & - \\
\hline Mahonia aquifolium & Shrub & $\mathrm{N}$ America & - & - & - & - & - & Dry shrub and forest communities \\
\hline Prunus serotina & Tree & N America & (6) & $\mathrm{x}$ & 5 & $\mathrm{x}$ & - & acidic oak forests \\
\hline Quercus rubra & Tree & N America & - & - & - & - & - & - \\
\hline Robinia pseudoacacia & Tree & N America & (5) & 4 & 4 & $\mathrm{x}$ & 8 & Dry shrub and forest communities, floodplain forests \\
\hline Solidago canadensis & Perennial herb & N America & 8 & 5 & $\mathrm{x}$ & $\mathrm{x}$ & 6 & Floodplain forests \\
\hline Symphoricarpos albus & Shrub & N America & - & - & - & - & - & Floodplain forests \\
\hline References & $\mathrm{E}, \mathrm{F}$ & $\mathrm{F}, \mathrm{K}, \mathrm{L}, \mathrm{O}$ & & & $\mathrm{B}, \mathrm{E}$ & & & $\mathrm{L}, \mathrm{O}$ \\
\hline
\end{tabular}

-: no data available; $\times$ : indifferent; ( ): value for tree seedlings and saplings; for further explanation of the indicator values see text.

those in the cluster group 13-23. The highest median in the clusters 3 and 11 with the value 4.5 indicates sites with the highest nitrogen availability for plants in the investigated pine forests compared with all other communities. Here, the species Arrhenatherum elatius, Brachypodium sylvaticum, Mycelis muralis, Rubus fruticosus agg., and Rubus idaeus (nitrogen value $\geq 6$ ) occur with high frequencies (Tab. II).

In general, the median of the indicator value for light $(\mathrm{L})$ is 6.0 (Fig. 2), thus showing relatively light growth conditions under the pine canopies. The cluster medians of the indicator value for continentality $(\mathrm{C})$ range between 3.0 and 5.0, indicating oceanic to sub-oceanic climate conditions. The medians of the indicator values for moisture (M) show the whole spectrum of pine forests growing on dry to wet sites. The medians mainly range between 4.0 and 5.0 (Fig. 2), which means not very dry sites [15]. The lowest values were recorded for cluster 1 and 13 . Both medians are significantly $(p<0.05)$ to highly significantly $(p<0.01)$ lower compared to the medians of all other clusters. These two communities are characterised by, e.g. Corynephorus canescens, Helichrysum arenarium, Polytrichum piliferum, Sedum maximum, and Spergula morisonii (indicator value for moisture $\leq 3$ ), which are typical for dry to very dry sandy soils. On the contrary, clusters 15 and 19 are the communities with the highest medians, highly significantly $(p<0.01)$ different from all other clusters. Here, Ledum palustre frequently occurs in both clusters and additionally only in cluster 19, Aulacomnium palustre, Eriophorum vaginatum, Sphagnum div. sp., and Vaccinium oxycoccus (moisture value $\geq 7$ ) occur.

The ecological range of the clusters or cluster groups is shown in Figure 3 as an ecogram (for the method see [14, 18]). In order to allow a comparison with the ecogram given for natural forest communities in Central Europe (Fig. 3A) by Ellenberg [14], the ecological site factors moisture and soil reaction are also shown for the differentiated clusters (Fig. 3B).

\subsection{Non-indigenous species and their site ecological range in pine forests}

On the basis of the investigated data set, the non-indigenous species Acer negundo, Amelanchier alnifolia, Campylopus introflexus, Conyza canadensis, Impatiens parviflora, Ligustrum vulgare, Mahonia aquifolium, Prunus serotina, Quercus rubra, Robinia pseudoacacia, Solidago canadensis, and Symphoricarpos albus are recorded in the pine forests (Tab. II). Thus, only about $5 \%$ of the taxa in the investigated pine forests of NE Germany are non-indigenous. These are mainly trees and shrubs with the exception of three herb species and one bryophyte. Most of the species were introduced from N America, few from Asia, S Europe, and the south hemisphere (Campylopus introflexus native to S America, S Africa, Australia, and New Zealand).

For most of the recorded non-indigenous species, a pronounced ecological site preference can be revealed. Thus, Acer negundo, Amelanchier alnifolia, Ligustrum vulgare, Mahonia aquifolium, Solidago canadensis, and Symphoricarpos albus only occur in clusters $1-12$, which are characterised by relatively high mean indicator values for soil reaction $(\mathrm{R})$ and nitrogen availability $(\mathrm{N})$. Additionally, all other non-indigenous vascular plant species (Conyza canadensis, Impatiens parviflora, Prunus serotina, Quercus rubra, and Robinia pseudoacacia) show their highest frequencies within this group and/ or in single clusters of this group. The bryophyte Campylopus introflexus however, is the only non-indigenous species, which tends to be more frequent in the more acid and nitrogen poor cluster group, in particular in cluster 13 (Cladonio-Pinetum Juraszek 1927).

Most of the recorded non-indigenous species occur in cluster 2. In this community, Acer negundo, Amelanchier alnifolia, Conyza canadensis, Ligustrum vulgare, Mahonia aquifolium, Prunus serotina, and Symphoricarpos albus reach the 
A: Central European Forest Communities

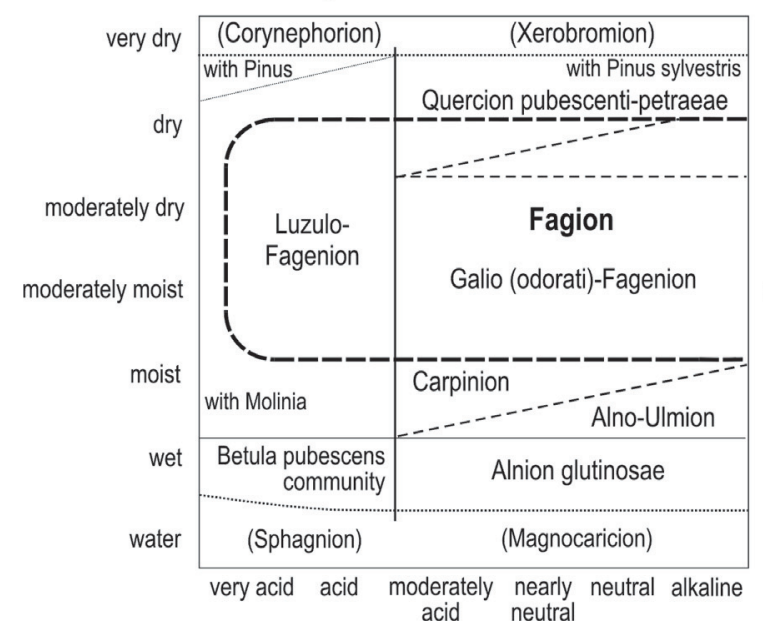

B: Community Types of Pine Forests in NE Germany

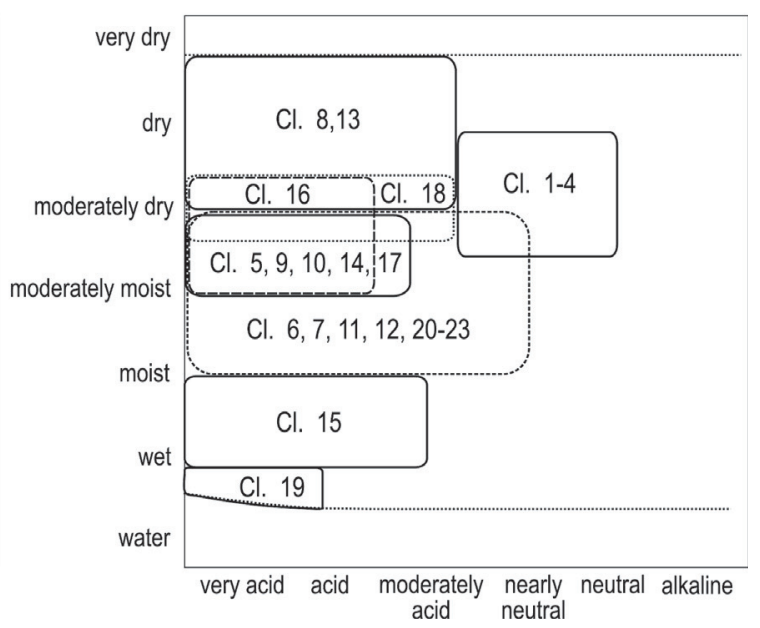

Figure 3. Ecogram of the pine forest community types in NE Germany (B) revealed by cluster analysis in comparison with the data given for natural woodland in Central Europe (A) by [14] along the soil moisture gradient and pH range.

highest frequencies compared to all other clusters. Solidago canadensis is most frequent in cluster 4 , which is characterised by the highest median (5; Fig. 2) of the indicator value for soil reaction $(\mathrm{R})$ and, with regard to the single vegetation plots of this cluster, medians of the indicator value for nitrogen supply $(\mathrm{N})$, which range from 4 to 6 (cluster median 4 ).

From the occurrence of non-indigenous species in different clusters, it can be concluded that Prunus serotina and Quercus rubra show the broadest ecological range. It has to be stated, that clusters 15 and 19 with the highest median for moisture (67 ) are free of non-indigenous species. Additionally, in cluster 17 with the lowest median for light (5.5) almost no non-indigenous species are present (with the exception of Quercus rubra with a frequency of only $2 \%$ ).

\section{DISCUSSION}

\subsection{Few non-indigenous species in the forests}

With only about $5 \%$ of the total species pool of the analysed data set, the number of recorded non-indigenous species in pine forests, which cover a broad ecological site range in NE Germany, is very low. This low number most probably will more or less increase, if other specific woodland sites like forest paths, edges, or forest springs will be taken into account. Alberternst and Nawrath [1] for example, observed the spread of the North American Lysichiton americanus on swampy forest sites in the Taunus region (W Germany). Dostálek [11] mapped non-indigenous plants like, for example, the East Asian Reynoutria japonica and the North American Rudbeckia laciniata along roads through a woodland area of the Orlické mountains in the Czech Republic. However, it is widely recognised that much less non-indigenous plant species are found in forests compared to the open landscape with its agricultural and settlement habitats [34]. On the basis of about 5100 vegetation plots, Kowarik [31] shows the percentages of non-indigenous plant species for different plant communities, occurring in the city of Berlin. The proportion of non-indigenous species is highest in short-lived plant communities (e.g. Fumario-Euphorbion Th. Müller in Görs 1966, Dauco-Melilotion Görs ex Oberd. et al. 1967, Sisymbrion R. Tx. et al. in R. Tx. 1950). On the contrary, lowest percentages of non-indigenous species are revealed for forest communities and the vegetation of mires and open waters. "Resistance" of ecosystems against invasive species are not considered responsible for the low numbers of non-indigenous species in forests, but limited propagule pressure and dispersal $[33,62]$.

The results obtained in this study on pine forests, correspond with the fact, that there are relatively few successful invaders also in other Central European forest types (see the comprehensive survey by Lohmeyer and Sukopp [40]). According to Lohmeyer and Sukopp [40], in particular natural floodplain forests and forests on dry habitats seem to be most susceptible to the establishment of non-indigenous species (Tab. II). One of the most successful invaders is Impatiens parviflora, native to E Asia, which can also be found in beech forests, naturally widespread in Central Europe [61].

\subsection{Ecological factors enhancing and limiting the occurrence of non-indigenous species in forests}

The cluster analysis and the site ecological assessment, applying Ellenberg indicator values reveal the fact that Pinus sylvestris dominates a large spectrum of sites in the German lowlands with regard to soil properties such as moisture, nutrient availability, and $\mathrm{pH}$. Within the site ecological range of the non-indigenous species in pine forests of NE Germany, three ecological groups can be distinguished: non-indigenous species, which occur:

(1) in nearly neutral, relatively nitrogen rich, and moderately moist habitats under oceanic climate conditions such as Acer negundo, Amelanchier alnifolia, Conyza canadensis, Impatiens parviflora, Ligustrum vulgare, Mahonia aquifolium, Robinia pseudoacacia, Solidago canadensis, and Symphoricarpos albus; 
(2) in acid to nearly neutral, nitrogen poor to relatively rich, and moderately moist habitats under oceanic and sub-oceanic climate conditions such as Prunus serotina and Quercus rubra, and

(3) in acid, very nitrogen poor, and dry habitats under sub-oceanic climate conditions such as Campylopus introflexus.

This corresponds quite well with the ecological indicator values given for these non-indigenous plant species by Ellenberg et al. [15] and Benkert et al. [4] for Central Europe (Tab. II; data not for all species available). One exception is Ligustrum vulgare which seems to grow not only on alkaline sites as indicated by the $\mathrm{R}$ value (8), but also occur on nearly neutral sites in pine forests.

As limiting ecological factors for the occurrence of nonindigenous species in NE German pine forests, in particular soil acidity, low nitrogen availability, and stagnating wetness could be identified. The clusters which correspond on the one hand to very acid and nitrogen poor conditions, e.g. sites of the Cladonio-Pinetum Juraszek 1927 and Leucobryo-Pinetum Matuszk. $1962[19,37]$ and on the other hand to very wet sites with the Ledo-Pinetum (Hueck 1929) R. Tx. 1955 [36] and Vaccinio uliginosi-Pinetum sylvestris Kleist 1929 [51] in general show only very low frequencies of non-indigenous vascular plants or even no non-indigenous species at all.

However, this could not be so clearly revealed for the nonindigenous tree species Prunus serotina and Quercus rubra from N America, which also occur on sites with relatively low soil reaction $(\mathrm{R})$ and nitrogen $(\mathrm{N})$ values (Tab. I and Fig. 2). Both species have been planted by forestry, e.g. for timber production $[30,58]$. Thus, taking also the relatively high frequencies of the two species in the single clusters of the cluster group 1-12 into account (Tab. I), it seems to be obvious, that these species can more easily establish themselves also on dry and acid sites when fostered by plantations. This has been also shown for the N American Douglas fir (Pseudotsuga menziesii), which spontaneously invades Central European forests as a consequence of forest plantations (e.g. [29]).

On the contrary, relatively high nutrient availability seems to enhance the establishment of non-indigenous species, which in particular is revealed by the high number and frequency of non-indigenous species in clusters $2-4$. The comparably high means of the $\mathrm{R}$ and $\mathrm{N}$ values of these clusters are the consequence of atmospheric depositions on naturally acid sites. Most of the vegetation samples within these clusters were taken from pine forests east of the city of Berlin [27] and from the Dübener Heide near the city of Bitterfeld, an industrial area in SachsenAnhalt [2]. In the latter region, a close relationship between alkaline atmospheric depositions and an increase of soil $\mathrm{pH}$ with the consequence of the alteration of vegetation in pine forests was revealed. In particular, the frequency of species, which commonly occur on disturbed sites and mesophytic meadows increased.

Accordingly, increased nutrient availability as well as human activity is considered to affect plant invasions positively in the investigated pine forest ecosystems. In particular, atmospheric nutrient depositions, a widespread phenomenon in Central Europe [71], could be a key factor. This is in accordance with the findings of Huennecke et al. [24], Hobbs and Huennecke [21], McIntyre and Lavorel [44], Stohlgren et al. [60],
Deutschewitz et al. [9], and Cassidy et al. [8], who point out a positive effect of habitat disturbance and nutrient availability on plant invasions.

\subsection{Reversibility and irreversibility of the plant invasions in pine forests}

According to Ellenberg [14], the natural occurrence of pine dominated forests in Central European woodland is extremely limited and restricted to very dry acid or calcareous soils and oligotrophic wet sites like mires (Fig. 3A). In Figure 3B, all clusters are arranged with regard to soil moisture (medians of the $\mathrm{M}$ indicator values) and $\mathrm{pH}$ range (medians of the $\mathrm{R}$ indicator values) in an ecogram. Consequently, clusters 8 and 13 (Cladonio-Pinetum) on dry and acid sandy sites and clusters 15 and 19 (Ledo-Pinetum and Vaccinio uliginosi-Pinetum) on wet and acid sites contain vegetation plots of natural pine forest communities. On the contrary, all other communities represented by the investigated pine stands in the NE German lowland are considered more or less anthropogenic (e.g. pine plantations) and/or are succession stages which naturally develop towards broad-leaved forests built up by beech (Fagus sylvatica), oak (Quercus petraea and Quercus robur), lime tree (Tilia cordata), hornbeam (Carpinus betulus), and others (e.g. $[42,70])$. This is reflected by the frequency of tree species (incl. spontaneous tree rejuvenation). In the stands of the CladonioPinetum, Ledo-Pinetum, and Vaccinio uliginosi-Pinetum, the very low frequencies or absence of Fagus sylvatica, Quercus spp., and other broad-leaved tree species were recorded. In contrast, oak is present with high frequencies of more than $90 \%$ in most of the other community types.

In conclusion, it is hypothesised that all those non-indigenous species with a high light demand such as Conyza canadensis, Ligustrum vulgare, and Solidago canadensis (see Tab. II for the Ellenberg indicator values) will not be able to grow in more or less dense broad-leaved forests. As the bryophyte Campylopus introflexus was recorded in the Cladonio-Pinetum (e.g. [38]), which is considered as a natural pine forest community, it is naturalised in these forests. Additionally, Impatiens parviflora, which is a relatively shade tolerant species, has been fully naturalised in Central European forests [40]. Following the hypothesis, that the light demanding non-indigenous plant species won't find suitable living conditions in natural mixed broad-leaved forests, it can be recommended to converse the anthropogenic pine forests in NE Germany towards more natural broad-leaved forests, in order to accelerate the reversion of these plant invasions. This forest conversion is in accordance with the general objective of Central European forestry to restore natural woodland [28, 49, 70], in particular on sites where at present anthropogenic coniferous forests occur.

Acknowledgements: We thank Ch. Berg, Th. Heinken, and K.H. Müller for making their digital data available. Also, we are indebted to H. Sukopp, W. Seidling, and two anonymous reviewers for their valuable comments on the manuscript, to W. Roloff for creating the figures, and to P. E. Herreid for improving our English. This study was financially supported by the German Federal Ministry of Research and Education (BMBF) within the research project FOREST (research program BIOTEAM, Fkz. 01 LM 0207). 


\section{REFERENCES}

[1] Alberternst B., Nawrath S., Lysichiton americanus in Central Europe - is there a chance to control the invasive species in the early phase of its naturalisation? BfN-Skripten 32 (2001) 37-38.

[2] Amarell U., Kiefernforste der Dübener Heide. Ursachen und Verlauf der Entstehung und Veränderung von Forstgesellschaften, Diss. Bot. 325 (2000) 1-246.

[3] Backhaus K., Erichson B., Plinke W., Schuchard-Ficher C., Weiber R., Multivariate Analysemethoden, 8th ed., Springer, Berlin, Heidelberg, New York, 1996.

[4] Benkert D., Erzberger P., Klawitter J., Linder W., Linke C., Schaepe A., Steinland M., Wiehle W., Liste der Moose von Brandenburg und Berlin mit Gefährdungsgraden, Verh. Bot. Ver. Berlin Brandenbg. 128 (1995) 1-70.

[5] Berg C., Dengler J., Abdank A., Die Pflanzengesellschaften Mecklenburg-Vorpommerns und ihre Gefährdung, Tables, Landesamt für Umwelt, Naturschutz u. Geologie Mecklenburg-Vorpommern (Ed.), Weissdorn, Jena, 2001.

[6] Braun-Blanquet J., Pflanzensoziologie. Grundzüge der Vegetationskunde, 3rd ed., Springer, Wien, 1964.

[7] Brosius G., Brosius F., SPSS. Base System and Professional Statistics, 1st ed., Int. Thomson Publ., Bonn, 1995.

[8] Cassidy T.M., Fownes J.H., Harrington R.A., Nitrogen limits an invasive perennial shrub in forest understory, Biol. Invas. 6 (2004) $113-121$.

[9] Deutschewitz K., Lausch A., Kühn I., Klotz S., Native and alien plant species richness in relation to spatial heterogeneity on a regional scale in Germany, Glob. Ecol. Biogeogr. 12 (2003) 299311.

[10] Diekmann M., Eilertsen O., Fremstad E., Lawesson J.E., Aude E., Beech forest communities in the Nordic countries - a multivariate analysis, Plant Ecol. 140 (1999) 203-220.

[11] Dostálek J., Changes in the distribution of synanthropic plants along roads in the territory of the Protected Landscape Area of the Orlické hory Mts., Příroda 10 (1997) 159-182.

[12] Dupré C., Diekmann M., Prediction of occurrence of vascular plants in deciduous forests of South Sweden by means of Ellenberg indicator values, Appl. Veg. Sci. 1 (1998) 139-150.

[13] Durka W., Ackermann W., SORT - Ein Computerprogramm zur Bearbeitung von floristischen und faunistischen Artentabellen, Natur u. Landsch. 68 (1993) 16-21.

[14] Ellenberg H., Vegetation ecology of Central Europe, Cambridge Univ. Press, Cambridge, 1988.

[15] Ellenberg H., Weber H.E., Düll R., Wirth V., Werner W., Paulissen D., Zeigerwerte von Pflanzen in Mitteleuropa, Scripta Geobot. 18 (1991) 1-247.

[16] Fitschen J., Gehölzflora, Quelle \& Meyer, Heidelberg, Wiesbaden, 1990.

[17] Frahm J.-P., Frey W., Moosflora, 3rd ed., Ulmer, Stuttgart, 1992.

[18] Härdtle W., v. Oheimb G., Friedel A., Meyer H., Westphal C., Relationship between $\mathrm{pH}$-values and nutrient availability in forest soils - the consequences for the use of ecograms in forest ecology, Flora 199 (2004) 134-142.

[19] Heinken T., Zippel E., Die Sand-Kiefernwälder (Dicrano-Pinion) im norddeutschen Tiefland: Syntaxonomische, standörtliche und geographische Gliederung, Tuexenia 19 (1999) 55-106.

[20] Hendel M., Klima, in: Liedke H., Marcinek J. (Eds.), Physische Geographie Deutschlands, 3rd ed., H. Haack Verlagsges., Gotha, 2002, pp. 14-119.
[21] Hobbs R.J., Huenneke L.F., Disturbance, diversity, and invasions: Implications for conservation, Conserv. Biol. 6 (1992) 324-337.

[22] Hofmann G., Wald, Klima, Fremdstoffeintrag. Ökologischer Wandel mit Konsequenzen für Waldbau und Naturschutz dargestellt am Gebiet der neuen Bundesländer Deutschlands, Angew. Landschaftsökol. 4 (1995) 165-189.

[23] Hofmeister H., Garve E., Lebensraum Acker, 2nd ed., Parey, Berlin, 1998.

[24] Huennecke L.F., Hamburg S.P., Koide R., Mooney H.A., Vitousek P.A., Effects of soil resources on plant invasion and community structure in Californian serpentine grassland, Ecol. 71 (1990) 478491.

[25] Jurko A., Kontris J., Phytocoenological and ecological characteristics of acacia-woods in the little Carpathians, Biológia 37 (1982) $67-74$.

[26] Jussy J.-H., Ranger J., Bienaimé S., Dambrine E., Effects of a clearcut on the in situ nitrogen mineralisation and the nitrogen cycle in a 67-year-Douglas-fir (Pseudotsuga menziesii (Mirb.) Franco) plantation, Ann. For. Sci. 61 (2003) 397-408.

[27] Klemm G., Ristow M., Floristisch-vegetationskundliche Untersuchung im NSG Wilhelmshagen-Woltersdorfer Dünenzug, Verh. Bot. Ver. Berlin Brandenbg. 128 (1995) 193-229.

[28] Klimo E., Hager H., Kulhavý J. (Eds.), Spruce monocultures in Central Europe - problems and prospects, European Forest Institute Proceedings 33 (2000) 1-208.

[29] Knoerzer D., Zur Naturverjüngung der Douglasie im Schwarzwald, Diss. Bot. 306 (1999) 1-283.

[30] Knoerzer D., Reif A., Fremdländische Baumarten in deutschen Wäldern. Fluch oder Segen? in: Kowarik I., Starfinger U. (Eds.), Biologische Invasionen: Herausforderung zum Handeln? Neobiota 1 (2002) 27-35.

[31] Kowarik I., Zum menschlichen Einfuß auf Flora und Vegetation, Landschaftsentwickl. u. Umweltforschg. 56 (1988), 1-280.

[32] Kowarik I., Einführung und Ausbreitung nichteinheimischer Gehölzarten in Berlin und Brandenburg und ihre Folgen für Flora und Vegetation. Ein Modell für die Freisetzung gentechnisch veränderter Organismen, Verh. Bot. Ver. Berlin Brandenbg 3 (1992) 1-188.

[33] Kowarik I., Human agency in biological invasions: secondary releases foster naturalisation and population expansion of alien plant species, Biol. Invas. 5 (2003) 293-312.

[34] Kowarik I., Biologische Invasionen: Neophyten und Neozoen in Mitteleuropa, Ulmer, Stuttgart, 2003.

[35] Kowarik I., Seidling W., Zeigerwertberechnung nach Ellenberg. Zu Problemen und Einschränkungen einer sinnvollen Methode, Landsch. u. Stadt 21 (1989) 132-143.

[36] Krausch H.-D., Die Pflanzengesellschaften des Stechlinsee-Gebietes, IV. Die Moore, Limnologica 6 (1968) 321-380.

[37] Krausch H.-D., Die Pflanzengesellschaften des Stechlinsee-Gebietes, V. Wälder, Hecken und Saumgesellschaften, Limnologica 7 (1970) 397-454.

[38] Kürschner H., Runge S., Vegetationskundliche Untersuchungen ausgewählter Binnendünen- und Talsandstandorte im DahmeSeengebiet (Brandenburg) und ihre Entwicklungspotentiale, Verh. Bot. Ver. Berlin Brandenbg. 130 (1997) 79-110.

[39] Lang G., Quartäre Vegetationsgeschichte Europas, Fischer, Jena, Stuttgart, New York, 1994.

[40] Lohmeyer W., Sukopp H., Agriophyten in der Vegetation Mitteleuropas, Schrreihe Veg. kd. 25 (1992) 1-185.

[41] Lozán J.L., Kausch H., Angewandte Statistik für Naturwissenschaftler, 2nd ed., Pareys Studientexte 74 (1998) 1-287. 
[42] Lust N., Maddelein D., Neirynck J., Nachtergale L., Management of uneven-aged mixed stands originating from regeneration under Scots pine cover, in: Olsthoorn A.F.M., Bartelink H.H., Gardiner J.J., Pretzsch H., Hekhuis H.J., Franc A. (Eds.), Management of mixed-species forest: silviculture and economics, IBN Sci. Contrib. 15 (1999) 199-207.

[43] Matuszkiewicz W., Zur Systematik der natürlichen Kiefernwälder des mittel- und osteuropäischen Flachlandes, Mitt. Florist-Soziol. Arbeitsgem. N.F. 9 (1962) 145-168.

[44] McIntyre S., Lavorel S., Predicting richness of native, rare, and exotic plants in response to habitat and disturbance variables across a variegated landscape, Conserv. Biol. 8 (1994) 521-531.

[45] Meisel-Jahn S., Die Kiefernforstgesellschaften des nordwestdeutschen Flachlandes, Angew. Pflanzensoz., Stolzenau/Weser 11 (1955) 1-126.

[46] Mühr B., Klimadiagramme, Retrieved from http:// www.imk.physik.uni. karlsruhe.de/ muehr/climate/Frame/diagramme. html on 15 January 2003, 2002.

[47] Oberdorfer E., Süddeutsche Pflanzengesellschaften, Teil IV: Wälder und Gebüsche, 2nd ed., Fischer, Jena, 1992.

[48] Oberdorfer E., Pflanzensoziologische Exkursionsflora für Deutschland und angrenzende Gebiete, 8th ed., Ulmer, Stuttgart, 2001.

[49] Olsthoorn A.F.M., Bartelink H.H., Gardiner J.J., Pretzsch H., Hekhuis H.J., Franc A., Management of mixed-species forest: silviculture and economics, IBN Sci. Contrib. 15 (1999) 1-389.

[50] Passarge H., Pflanzengesellschaften des nordostdeutschen Flachlandes, I. Pflanzensoziologie 13 (1964) 1-324.

[51] Pott R., Die Pflanzengesellschaften Deutschlands, 2nd ed., Ulmer, Stuttgart, 1995.

[52] Pyšek P., Factors affecting the diversity of flora and vegetation in central European settlements, Vegetatio 106 (1993) 89-100.

[53] Sachs L., Angewandte Statistik, 9th ed., Springer, Heidelberg, New York, 1999.

[54] Sandlund O.T., Schei P.J., Viken A., Proceedings of the Norway/ UN conference on alien species, Directorate for Nature Management and Norwegian Institute for Nature Research, Trondheim, 1996.

[55] Scamoni A., Gedanken über die Verbreitung der Kiefer im Tiefland, Forstarchiv 59 (1988) 173-180.

[56] Schepker H., Kowarik I., Garve E., Verwilderungen nordamerikanischer Kultur-Heidelbeeren (Vaccinium subg. Cyanococcus) in Niedersachsen und deren Einschätzung aus Naturschutzsicht, Natur u. Landschaft 72 (1997) 346-351.

[57] Schmidt R., Böden, in: Liedke H., Marcinek J. (Eds.), Physische Geographie Deutschlands, 3rd ed., H. Haack Verlagsges., Gotha, 2002, pp. 198-218.
[58] Starfinger U., Die Einbürgerung der Spätblühenden Traubenkirsche (Prunus serotina Ehrh.) in Mitteleuropa, Landschaftsentwickl. u. Umweltforsch. 69 (1990) 1-119.

[59] Starfinger U., Introduction and naturalization of Prunus serotina in Central Europe, in: Brock J.H., Wade M., Pyšek P., Green D. (Eds.) Plant invasions: Studies from North America and Europe, Backhuys Publ., Leiden, The Netherlands, 1997, pp. 161-171.

[60] Stohlgren T.J., Binkley D., Chong G.W., Kalkhan M.A., Schell L.D., Bull K.A., Otsuki J., Newman G., Bashkin M., Son Y., Exotic plant species invade hot spots of native plant diversity, Ecol. Monogr. 69 (1999) 25-46.

[61] Trepl L., Über Impatiens parviflora DC. Agriophyt in Mitteleuropa, Diss. Bot. 73 (1984) 1-400.

[62] Trepl L., Zum Problem der Resistenz von Pflanzengesellschaften gegen biologische Invasionen, Verh. Berl. Bot. Ver. 8 (1990) 195230.

[63] U.S. Congress, O.T.A., Harmful non-indigenous species in the United States, US Government Printing Office, Washington DC, 1993.

[64] Vitousek P.M., D'Antonio C.M., Loope L.L., Rejmanek M., Westbrooks R., Introduced species: a significant component of human-caused global change, N. Z. J. Ecol. 21 (1997) 1-16.

[65] Volz H., Otte A., Occurrence and spreading ability of Lupinus polyphyllus Lindl. in the Hochrhoen, in: Kowarik I., Starfinger U. (Eds.), Biological invasions in Germany. A challenge to act? BfNSkripten 32 (2001) 97-98.

[66] Wildi O., Keller W., Kuhn N., Krüsi B.O., Schütz M., Wohlgemuth T., Revision der Waldgesellschaften der Schweiz: Die Analyse einer nicht-systematischen Datenbasis, in: Zerbe S. (Ed.), Vegetationsökologie mitteleuropäischer Wälder, Landschaftsentwickl. u. Umweltforsch. 104 (1996) 37-48.

[67] Wirth V., Flechtenflora, 2nd ed., Ulmer, Stuttgart, 1995.

[68] Wisskirchen R., Haeupler H., Standardliste der Farn- und Blütenpflanzen Deutschlands, Ulmer, Stuttgart, 1998.

[69] Zerbe S., Die Wald- und Forstgesellschaften des Spessarts mit Vorschlägen zu deren zukünftigen Entwicklung, Mitt. Naturwiss. Mus. Aschaffenbg. 19 (1999) 1-354.

[70] Zerbe S., Restoration of natural broad-leaved woodland in Central Europe on sites with coniferous forest plantations, For. Ecol. Manage. 167 (2002) 27-42.

[71] Zerbe S., Brande A., Woodland degradation and regeneration in Central Europe - a case study in NE Germany, Phytocoenologia 33 (2003) 683-700.

[72] Zerbe S., Maurer U., Schmitz S., Sukopp H., Biodiversity in Berlin and its potential for nature conservation, Landsc. Urban. Plan. 62 (2003) 139-148. 